\title{
Stability of continuous-time quantum filters with measurement imperfections
}

\author{
Hadis Amini * Clément Pellegrini ${ }^{\dagger} \quad$ Pierre Rouchon ${ }^{\ddagger}$
}

December 2, 2013

\begin{abstract}
The fidelity between the state of a continuously observed quantum system and the state of its associated quantum filter, is shown to be always a submartingale. The observed system is assumed to be governed by a continuous-time Stochastic Master Equation (SME), driven simultaneously by Wiener and Poisson processes and that takes into account incompleteness and errors in measurements. This stability result is the continuous-time counterpart of a similar stability result already established for discrete-time quantum systems and where the measurement imperfections are modeled by a left stochastic matrix.
\end{abstract}

Keywords. Quantum filtering, stability, continuous-time stochastic master equations, Wiener process, Poisson process, quantum trajectories, measurement errors.

\section{Introduction}

Since the work of Davies [14], the time evolution of the state (density operator) $\rho_{t}$ at time $t$ of an observed quantum system can be described by a Stochastic Master Equation (SME) taking into account the back-action of the measurements on $\rho_{t}$. Such SMEs (see [5] for a modern exposure) have been the starting point of the seminal contributions of Belavkin to quantum filtering and control $[7,8,4,9]$. Quantum filters are used to get an estimate $\rho_{t}^{e}$ of $\rho_{t}$ based on an initial guess $\rho_{0}^{e}$ of $\rho_{0}$ and on the measurement outcomes between 0 and $t$. Quantum filtering is related to quantum trajectories $[13,12]$ and their original motivations for Monte-Carlo simulations. Roughly speaking, quantum filtering replaces the random numbers used at each time-step of a Monte-Carlo simulation by the measurement outcomes to update the estimate $\rho_{t}^{e}$. An important practical issue deals with the convergence: does $\rho_{t}^{e}$ converge towards $\rho_{t}$ when $t$ tends to $+\infty$, even if $\rho_{0}^{e} \neq \rho_{0}$ ? Few convergence results are available up to now, except the sufficient conditions established in [24, 25] for diffusive SMEs. Particular results for quantum non demolition indirect measurements have been established in $[6,10]$ As far as we know, general and checkable necessary and sufficient convergence conditions do not exist yet.

\footnotetext{
*Edward L. Ginzton Laboratory, Stanford University, Stanford, CA 94305, USA, nhamini@stanford.edu.

†Institut de Mathématiques, IMT, Université de Toulouse (UMR 5219), 31062 Toulouse, Cedex 9, France, clement.pellegrini@math.univ-toulouse.fr.

${ }^{\ddagger}$ Centre Automatique et Systèmes, Mines ParisTech, 60 Bd Saint Michel, 75272 Paris cedex 06, France, pierre.rouchon@mines-paristech.fr.
} 
In this paper we do not investigate directly convergence issues. We focus on stability issues to ensure the absence of time divergence. We prove in Theorem 5 that for a large class of continuous-time SMEs driven simultaneously by Wiener and Poisson processes, associated quantum filters are stable: the fidelity between $\rho$ and its estimate $\rho^{e}$,

$$
F\left(\rho, \rho^{e}\right)=\operatorname{Tr}^{2}\left(\sqrt{\sqrt{\rho} \rho^{e} \sqrt{\rho}}\right)
$$

is a sub-martingale. In the Wiener case, Theorem 5 shows that, whatever the Hamiltonian $H$, the measurement operators $V_{\nu}$ and the detection efficiencies $\left.\bar{\eta}_{\nu} \in \mid 0,1\right]$ are, the fidelity $F\left(\rho_{t}, \rho_{t}^{e}\right)$ is a sub-martingale where $\rho_{t}$ obeys the following diffusive SME

$$
\begin{aligned}
d \rho_{t}=-i\left[H, \rho_{t}\right] d t+\left(\sum_{\nu} V_{\nu} \rho_{t} V_{\nu}^{\dagger}\right. & \left.-\frac{1}{2}\left(V_{\nu}^{\dagger} V_{\nu} \rho_{t}+\rho_{t} V_{\nu}^{\dagger} V_{\nu}\right)\right) d t \\
& +\sum_{\nu} \sqrt{\bar{\eta}_{\nu}}\left(V_{\nu} \rho_{t}+\rho_{t} V_{\nu}^{\dagger}-\operatorname{Tr}\left(\left(V_{\nu}+V_{\nu}^{\dagger}\right) \rho_{t}\right) \rho_{t}\right) d W_{\nu}(t)
\end{aligned}
$$

driven by the Wiener processes $W_{\nu}$, and where the estimate $\rho_{t}^{e}$ obeys the following non-linear stochastic equation

$$
\begin{aligned}
& d \rho_{t}^{e}=-i\left[H, \rho_{t}^{e}\right] d t+\left(\sum_{\nu} V_{\nu} \rho_{t}^{e} V_{\nu}^{\dagger}-\frac{1}{2}\left(V_{\nu}^{\dagger} V_{\nu} \rho_{t}^{e}+\rho_{t}^{e} V_{\nu}^{\dagger} V_{\nu}\right)\right) d t \\
& \quad+\sum_{\nu} \sqrt{\bar{\eta}_{\nu}}\left(V_{\nu} \rho_{t}^{e}+\rho_{t}^{e} V_{\nu}^{\dagger}-\operatorname{Tr}\left(\left(V_{\nu}+V_{\nu}^{\dagger}\right) \rho_{t}^{e}\right) \rho_{t}^{e}\right)\left(d y_{\nu}(t)-\sqrt{\bar{\eta}_{\nu}} \operatorname{Tr}\left(\left(V_{\nu}+V_{\nu}^{\dagger}\right) \rho_{t}^{e}\right) d t\right) .
\end{aligned}
$$

driven by the measures $d y_{\nu}(t)=d W_{\nu}(t)+\sqrt{\bar{\eta}_{\nu}} \operatorname{Tr}\left(\left(V_{\nu}+V_{\nu}^{\dagger}\right) \rho_{t}\right) d t$ and initialized to any density matrix $\rho_{0}^{e}$. In the Poisson case, Theorem 5 ensures, as for the Wiener case, the stability of the quantum filtering process. Additionally, it provides a new kind of SMEs taking into account incompleteness and errors in the jump detections:

$$
\begin{aligned}
d \rho_{t} & =-i\left[H, \rho_{t}\right] d t+\left(\sum_{\mu} V_{\mu} \rho_{t} V_{\mu}^{\dagger}-\frac{1}{2}\left(V_{\mu}^{\dagger} V_{\mu} \rho_{t}+\rho_{t} V_{\mu}^{\dagger} V_{\mu}\right)\right) d t \\
& +\sum_{\mu}\left(\frac{\bar{\theta}_{\mu} \rho_{t}+\sum_{\nu} \bar{\eta}_{\mu, \nu} V_{\nu} \rho_{t} V_{\nu}^{\dagger}}{\bar{\theta}_{\mu}+\sum_{\nu} \bar{\eta}_{\mu, \nu} \operatorname{Tr}\left(V_{\nu} \rho_{t} V_{\nu}^{\dagger}\right)}-\rho_{t}\right)\left(d N_{\mu}(t)-\left(\bar{\theta}_{\mu}+\sum_{\nu} \bar{\eta}_{\mu, \nu} \operatorname{Tr}\left(V_{\nu} \rho_{t} V_{\nu}^{\dagger}\right)\right) d t\right)
\end{aligned}
$$

driven by the the Poisson processes $N_{\mu}(t)$ with $\left\langle d N_{\mu}(t)\right\rangle=\left(\bar{\theta}_{\mu}+\sum_{\nu} \bar{\eta}_{\mu, \nu} \operatorname{Tr}\left(V_{\nu} \rho_{t} V_{\nu}^{\dagger}\right)\right) d t$, where the detection imperfections are modeled through the parameters $\bar{\theta}_{\mu} \geq 0$ and $\bar{\eta}_{\mu, \nu} \geq 0$ with $\sum_{\mu} \bar{\eta}_{\mu, \nu} \leq 1$.

The proof of Theorem 5 is based on discrete-time approximations of continuous-time SMEs. Such approximations have already been investigated in $[3,15,17,18]$. They rely on indirect measurements, originally introduced in [11] and well explained with suggestive physical systems in [16, 26]. For discrete-time SMEs, it is proved in [21, 23] that the fidelity between the quantum state and its estimate is always a submartingale. Theorem 5 is obtained by passing to the limit from discrete to continuous time. The fidelity between the quantum 
state and its estimate remains a submartingale. The obtained continuous-time SMEs are slightly more general than the ones usually encountered in the literature. Such SMEs could be of some interest to derive quantum filters taking into account a larger class of incompleteness and errors in measurements and jump detections.

This paper is structured in two main sections. Section 2 is devoted to Theorem 2, a restrictive version of Theorem 5 to the diffusive SMEs with perfect measurements. For this simplified but representative case, the discrete-time approximation is presented and the passage to the continuous-time limit is detailed during the proof of Proposition 1. Section 3 is devoted to Theorem 5 and fully exploits the tools and methods developed in Section 2. We consider SMEs driven simultaneously by Poisson and Wiener processes. We recall first the structure, described in [23] and based on a left stochastic matrix, of discrete-time SMEs associated to imperfect measurements. We apply on the discrete-time approximations such left stochastic matrix modeling of imperfections and errors. Then we take, thanks to Theorem 4, the limit to get the continuous-time SMEs and its associated quantum filters with imperfections. Their structures are more general than the usual ones encountered in the literature. This leads to Theorem 5 ensuring the stability of the obtained quantum filters. Section 4 is a short conclusion proposing some connection with Petz characterization of monotone metrics on matrix spaces.

Some intermediate and partial results related to Theorems 5 can be founded in $[2,1]$.

\section{Perfect measurements}

Let us start this section by presenting the jump-diffusive SMEs describing the evolutions of quantum state $\rho_{t}$ and its estimate $\rho_{t}^{e}$.

\subsection{Continuous-time filters}

We consider quantum systems of finite dimensions $1<N<\infty$. The state space of such a system is given by the set of density matrices

$$
\mathcal{D}:=\left\{\rho \in \mathbb{C}^{N \times N} \mid \quad \rho=\rho^{\dagger}, \quad \operatorname{Tr}(\rho)=1, \quad \rho \geq 0\right\} .
$$

Formally a real quantum trajectory $\rho \in \mathcal{D}$ in Schrödinger picture can be described by the following SME (cf. $[8,9,5])$

$$
d \rho_{t}=\left(-i\left[H, \rho_{t}\right]+\sum_{\xi} \mathcal{L}_{\xi}\left(\rho_{t}\right)\right) d t+\sum_{\nu} \Lambda_{\nu}\left(\rho_{t}\right) d W_{\nu}(t)+\sum_{\mu} \Upsilon_{\mu}\left(\rho_{t}\right)\left(d N_{\mu}(t)-\operatorname{Tr}\left(V_{\mu} \rho_{t} V_{\mu}^{\dagger}\right) d t\right),
$$

where

- the notation $[A, B]$ refers to $A B-B A$;

- $H=H^{\dagger}$ is a Hermitian operator corresponding to the total Hamiltonian of the system;

- $d N_{\mu}$ are the Poisson processes with $\mu \in\left\{1, \cdots, m_{P}\right\}$ and $d W_{\nu}$ are the Wiener processes with $\nu \in\left\{m_{P}+1, \cdots, m_{P}+m_{W}\right\}$; 
- The Lindblad superoperator $\mathcal{L}_{\xi}\left(\xi \in\left\{1, \ldots, m_{W}+m_{P}\right\}\right)$ is defined by

$$
\mathcal{L}_{\xi}(\rho):=V_{\xi} \rho V_{\xi}^{\dagger}-\frac{1}{2}\left(V_{\xi}^{\dagger} V_{\xi} \rho+\rho V_{\xi}^{\dagger} V_{\xi}\right)
$$

where $V_{\xi}$ is an arbitrary matrix which determines the measurement process (typically the coupling to the probe field for quantum optic systems);

- The superoperators $\Lambda_{\nu}$ and $\Upsilon_{\mu}$ are defined respectively by

$$
\Lambda_{\nu}(\rho):=V_{\nu} \rho+\rho V_{\nu}^{\dagger}-\operatorname{Tr}\left(\left(V_{\nu}+V_{\nu}^{\dagger}\right) \rho\right) \rho \quad \text { and } \quad \Upsilon_{\mu}(\rho):=\frac{V_{\mu} \rho V_{\mu}^{\dagger}}{\operatorname{Tr}\left(V_{\mu} \rho V_{\mu}^{\dagger}\right)}-\rho
$$

- The measurement outcomes are $d N_{\mu}$ and $d y_{\nu}$ where

$$
N_{\mu}(t)-\int_{0}^{t} \operatorname{Tr}\left(V_{\mu} \rho_{s} V_{\mu}^{\dagger}\right) d s \quad \text { is a martingale and } \quad d y_{\nu}(t)=d W_{\nu}(t)+\operatorname{Tr}\left(V_{\nu} \rho_{t} V_{\nu}^{\dagger}\right) d t
$$

All the developments remain valid when $\mathrm{H}$ and $V_{\xi}$ are deterministic time-varying matrices. For clarity sake, we do not recall below such possible time dependence.

In this paper, the notation $\rho^{e}$ corresponds to the estimate filter associated to the filter $\rho$. This estimate filter is provided from the measurement outcomes $d y_{\nu}$ and $d N_{\mu}$ and depends on the real quantum trajectory $\rho$ via the measurement outcomes (4). It has the following expression derived from (2) where $d W_{\nu}(t)$ is replaced by $d y_{\nu}(t)-\operatorname{Tr}\left(V_{\nu} \rho_{t}^{e} V_{\nu}^{\dagger}\right) d t$ (see e.g., [9, $5]):$

$$
\begin{aligned}
d \rho_{t}^{e}=\left(-i\left[H, \rho_{t}^{e}\right]+\sum_{\xi} \mathcal{L}_{\xi}\left(\rho_{t}^{e}\right)\right) d t+\sum_{\nu} \Lambda_{\nu}\left(\rho_{t}^{e}\right)\left(d y_{\nu}(t)-\operatorname{Tr}\left(V_{\nu} \rho_{t}^{e} V_{\nu}^{\dagger}\right) d t\right) & \\
& +\sum_{\mu} \Upsilon_{\mu}\left(\rho_{t}^{e}\right)\left(d N_{\mu}(t)-\operatorname{Tr}\left(V_{\mu} \rho_{t}^{e} V_{\mu}^{\dagger}\right) d t\right)
\end{aligned}
$$

When $\rho_{0}^{e} \neq \rho_{0}, \rho_{t}^{e}$ and $\rho_{t}$ do not coincide in general. However, we will see that the fidelity between $\rho_{t}^{e}$ and $\rho_{t}$ is a sub-martingale.

\subsection{Discrete-time filters}

First let us briefly remind the model of quantum repeated indirect measurement approach. The physics underlying such approach is well explained in $[16,26]$. In $[3,15,19]$, it was rigorously shown that such discrete-time approximations associated to the real state converges to the continuous model described in Equation (2).

\subsubsection{Quantum repeated measurement approach}

We consider the setup of quantum repeated interaction of $\mathcal{H}$ (describing the Hilbert space of the system state) with an infinite chain $\bigotimes_{k} \mathcal{K}_{k}$ (describing the Hilbert space of the environment) with $\mathcal{K}_{k}=\mathcal{K}$ for all $k$. More precisely, the first copy $\mathcal{K}_{1}=\mathcal{K}$ interacts with $\mathcal{H}$ during a time $\delta$ and then disappear. Next, the second copy $\mathcal{K}_{2}$ comes to interact with $\mathcal{H}$ and so on. 
This setting is coupled with indirect measurement, that is, after each interaction between $\mathcal{H}$ and $\mathcal{K}$, a measurement of an observable of $\mathcal{K}$ is performed.

Here, we assume that $\mathcal{H}=\mathbb{C}^{N}$ and the environment (meter system) is composed of $m_{P}+m_{W}$ qubits, so $\mathcal{K}=\left(\mathbb{C}^{2}\right)^{\otimes m_{P}+m_{W}}$. Also, take $|\psi\rangle$ as the initial state of the system and $|G\rangle$ as the initial state of the qubits' meter which is defined as the ground state of the meter system: all qubits in the ground state $|g\rangle$. As a result, the initial state of the system coupled to its environment is described by $|\psi\rangle \otimes|G\rangle$.

For $\delta=1 / n$ with $n$ large, assume that the Schrödinger evolution between time 0 to time $1 / n$ is given by

$$
H_{\mathrm{tot}}=H \otimes I+\sqrt{n} \sum_{\xi}\left(i V_{\xi} \otimes \sigma_{+\xi}-i V_{\xi}^{\dagger} \otimes \sigma_{-\xi}\right)
$$

where

- $H$ is the Hamiltonian of the system used in (2) and the operators $V_{\xi}$ are those appearing in the Lindblad superoperators (3)

- $\sigma_{+_{\xi}}=(|e\rangle\langle g|)_{\xi}$ and $\sigma_{-\xi}=\sigma_{+_{\xi}}^{\dagger}=(|g\rangle\langle e|)_{\xi}$ where the notation $(A)_{\xi}$ is an operator $\mathcal{K}$ defined by $(A)_{\xi}=\bigotimes_{1 \leq i<\xi} I \otimes A \otimes \bigotimes_{\xi<i \leq m_{W}+m_{P}} I$. Note that $\sigma_{-\xi}|G\rangle=0$.

In the sequel, symbol $\otimes$ will be remove for compact formulae.

Take the observables $X_{\nu}=(|g\rangle\langle e|)_{\nu}+(|e\rangle\langle g|)_{\nu}$ for $\nu \in\left\{m_{P}+1, \cdots, m_{W}+m_{P}\right\}$ and $Z_{\mu}=(|e\rangle\langle e|)_{\mu}-(|g\rangle\langle g|)_{\mu}$ for $\mu \in\left\{1, \cdots, m_{P}\right\}$. For $n$ large, the measurement of all qubits at final time $t=1 / n$ according to the observables $X_{\nu}$ and $Z_{\mu}$, yields an approximation for the Wiener and Poisson processes, respectively. This results from a development versus $1 / n$ of the measurement operators associated to the associated discrete-time stochastic evolution.

We have $e^{-i H_{\text {tot }} / n}=\mathbb{I}-\frac{i}{n} H_{\text {tot }}-\frac{1}{2 n^{2}} H_{\text {tot }}^{2}+\mathcal{O}\left(1 / n^{3 / 2}\right)$, where $\mathbb{I}$ is the identity operator. Now replacing $H_{\text {tot }}$ by its expression given in (6), we find

$$
e^{-i H_{\mathrm{tot}} / n} \approx \mathbb{I}+\frac{1}{\sqrt{n}} \sum_{\xi}\left(V_{\xi} \sigma_{+\xi}-V_{\xi}^{\dagger} \sigma_{-\xi}\right)-\frac{i}{n} H-\frac{1}{2 n} \sum_{\xi}\left(V_{\xi}^{\dagger} V_{\xi} P_{g_{\xi}}+V_{\xi} V_{\xi}^{\dagger} P_{e_{\xi}}\right),
$$

where $\approx$ means up to $\mathcal{O}\left(1 / n^{3 / 2}\right)$ terms. Here, $P_{g_{\xi}}=(|g\rangle\langle g|)_{\xi}$ and $P_{e_{\xi}}=(|e\rangle\langle e|)_{\xi}$. Note that

$$
P_{g_{\xi}}|G\rangle=|G\rangle \quad \text { and } \quad P_{e_{\xi}}|G\rangle=0 .
$$

Thus the system coupled to its environment evolves as follows

$$
e^{-i H_{\mathrm{tot}} / n}|\psi\rangle \otimes|G\rangle \approx\left(\mathbb{I}-\frac{i}{n} H-\frac{1}{2 n} \sum_{\xi} V_{\xi}^{\dagger} V_{\xi}\right)|\psi\rangle \otimes|G\rangle+\frac{1}{\sqrt{n}} \sum_{\xi} V_{\xi}|\psi\rangle \otimes\left|G_{\xi}\right\rangle .
$$

Let us consider the qubits measurements. The measurement outcomes for $X_{\nu}$ are stored in $x_{\nu-m_{P}} \in\{-1,1\}, x=\left(x_{\nu-m_{P}}\right) \in\{-1,1\}^{m_{W}}$, and those of $Z_{\mu}$ in $z_{\mu} \in\{0,1\}, z=\left(z_{\mu}\right) \in$ $\{0,1\}^{m_{P}}$, as follows (all these observables commute):

- if during the measure of $X_{\nu}$, the corresponding qubit collapses to $(|g\rangle+|e\rangle) / \sqrt{2}$ (resp. $(|g\rangle-|e\rangle) / \sqrt{2})$ set $x_{\nu-m_{P}}=+1$ (resp. $\left.x_{\nu-m_{P}}=-1\right)$. 
- if during the measure of $Z_{\mu}$, the corresponding qubit collapses to $|g\rangle$ (resp.|e〉), set $z_{\mu}=0\left(\right.$ resp. $\left.z_{\mu}=1\right)$.

The probability to get, for two different $\mu$ and $\mu^{\prime}, z_{\mu}=z_{\mu^{\prime}}=1$, is in order of $\mathcal{O}\left(1 / n^{3 / 2}\right)$. Thus for $z$, we need only consider the following cases: either all $z_{\mu}$ are equal to 0 or only a single one is equal to 1 , the other ones being 0 .

Consider the measurement outcomes $z=\left(z_{\mu}\right)$ and $x=\left(x_{\nu-m_{P}}\right)$. The associated wave packet collapse of $e^{-i H_{\text {tot }} / n}|\psi\rangle \otimes|G\rangle$ yields the un-normalized state $M_{x, z}|\psi\rangle \otimes|x, z\rangle$, where the measurement operator is denoted by $M_{x, z}$ and where $|x, z\rangle$ is the normalized state of the qubits characterized by $X_{\nu}|x, z\rangle=x_{\nu-m_{P}}|x, z\rangle$ and $Z_{\mu}|x, z\rangle=\left(2 z_{\mu}-1\right)|x, z\rangle$. We have to consider two situations: either $\sum z_{\mu}=0$ denoted by $z=0$ or $\sum_{\mu} z_{\mu}=1$. When $z_{\mu}=0$ for all $\mu$, some computations yield

$$
M_{x, 0} \approx\left(\prod_{\nu} \frac{1}{\sqrt{2}}\right)\left(\mathbb{I}-\frac{i}{n} H-\frac{1}{2 n} \sum_{\xi} V_{\xi}^{\dagger} V_{\xi}+\frac{1}{\sqrt{n}} \sum_{\nu} x_{\nu-m_{P}} V_{\nu}\right) .
$$

When $z_{\mu}=1$ and $z_{\mu^{\prime}}=0$ for all $\mu^{\prime} \neq \mu$, similar computations give

$$
M_{x, \mu} \approx\left(\prod_{\nu} \frac{1}{\sqrt{2}}\right) \frac{V_{\mu}}{\sqrt{n}}
$$

where we have denoted $M_{x, z}$ by $M_{x, \mu}$ for such $z$.

For an arbitrary state $\rho$ at $t=0$, not necessarily pure as $\rho=|\psi\rangle\langle\psi|$, the state $\rho_{1}$ at time $t=1 / n$ is given by

$$
\rho_{1}=\frac{M_{x, z} \rho M_{x, z}^{\dagger}}{p_{x, z}(\rho)}
$$

which happens with probability $p_{x, z}(\rho)=\operatorname{Tr}\left(M_{x, z} \rho M_{x, z}^{\dagger}\right)$.

The expression of $\rho_{1}$ is obtained by neglecting the terms of orders strictly greater than 1 versus $1 / n$. Some usual calculations yield

$$
\begin{aligned}
& M_{x, 0} \rho M_{x, 0}^{\dagger}= \\
& \quad c_{W}\left(\rho+\frac{1}{\sqrt{n}} \sum_{\nu} x_{\nu-m_{P}}\left(V_{\nu} \rho+\rho V_{\nu}^{\dagger}\right)+\frac{1}{n}\left(-i[H, \rho]-\sum_{\mu} \frac{\left\{\rho, V_{\mu}^{\dagger} V_{\mu}\right\}}{2}+\sum_{\nu} \mathcal{L}_{\nu}(\rho)\right)\right)
\end{aligned}
$$

with probability

$$
p_{x, 0}(\rho)=c_{W}\left(1+\frac{1}{\sqrt{n}} \sum_{\nu} x_{\nu-m_{P}} \operatorname{Tr}\left(\left(V_{\nu}+V_{\nu}^{\dagger}\right) \rho\right)-\frac{1}{n} \sum_{\mu} \operatorname{Tr}\left(\rho V_{\mu}^{\dagger} V_{\mu}\right)\right) .
$$

The notation $\{A, B\}$ used in (10) corresponds to $A B+B A$ and $c_{W}=\left(\frac{1}{2}\right)^{m_{W}}$.

Similarly, we have

$$
M_{x, \mu} \rho M_{x, \mu}^{\dagger}=\frac{c_{W}}{n} V_{\mu} \rho V_{\mu}^{\dagger}, \quad \text { with probability } \quad p_{x, \mu}(\rho)=\frac{c_{W}}{n} \operatorname{Tr}\left(V_{\mu} \rho V_{\mu}^{\dagger}\right) .
$$


Now we can obtain the asymptotic description of the transition for all possible observations. Indeed, the expression of the whole quantum trajectory $\rho_{k}$ can be obtained by replacing $\rho_{1}$ by $\rho_{k+1}$ and $\rho$ by $\rho_{k}$ in Equation (9).

As a conclusion, we find

$$
\rho_{k+1}=\frac{M_{x, \mu} \rho_{k} M_{x, \mu}^{\dagger}}{\operatorname{Tr}\left(M_{x, \mu} \rho_{k} M_{x, \mu}^{\dagger}\right)} \quad \text { with probability } \quad p_{x, \mu}\left(\rho_{k}\right)
$$

where $p_{x, \mu}\left(\rho_{k}\right)$ is given by $(12,11)$.

\subsubsection{Stability with respect to initial condition}

Consider the Markov chain (13). Assume that we do not know precisely the initial state $\rho_{0}$ and we have at our disposal an estimate $\rho_{0}^{e}$. Assume also that we know the measurement result at step $k\left(x_{k}, \mu_{k}\right) \in\{-1,1\}^{m_{W}} \times\{0,1\}$. It is then natural to consider the following recursive update of our estimation $\rho_{k+1}^{e}$ using the knowledge of measurement result at step $k$ and the previous estimate $\rho_{k}^{e}$ (see e.g., [26]):

$$
\rho_{k+1}^{e}=\frac{M_{x_{k}, \mu_{k}} \rho_{k}^{e} M_{x_{k}, \mu_{k}}^{\dagger}}{\operatorname{Tr}\left(M_{x_{k}, \mu_{k}} \rho_{k}^{e} M_{x_{k}, \mu_{k}}^{\dagger}\right)}
$$

Note that the probability $p_{x_{k}, \mu_{k}}\left(\rho_{k}\right)$ given by $(11,12)$ to get $\left(x_{k}, \mu_{k}\right)$ depends on the hidden state $\rho_{k}$ and not on $\rho_{k}^{e}$.

Remark 1. Let us stress that the above description is not always valid. Indeed, the normalization $\operatorname{Tr}\left(M_{x_{k}, \mu_{k}} \rho_{k}^{e} M_{x_{k}, \mu_{k}}^{\dagger}\right)$ can vanish and the formula (15) is then not defined. This problem does not appear when describing the true evolution since the normalization describing the true state corresponds to the probability of apparition (then if this vanishes this means that the corresponding state can not appear). This problem of non-definition for the discrete-time estimate filter is related to the problem underlined in the definition of (5). In general, this question has been taken into account in $[21,23]$ for the discrete-time filter. In our context focusing on asymptotic evolution, such a problem will not appear.

Theorem 1 ([21]). Consider any arbitrary Markov chain $\left(\rho_{k}, \rho_{k}^{e}\right)$ satisfying respectively Equations (13) and (15):

$$
\left(\rho_{k+1}, \rho_{k+1}^{e}\right)=\left(\frac{M_{x, \mu} \rho_{k} M_{x, \mu}^{\dagger}}{\operatorname{Tr}\left(M_{x, \mu} \rho_{k} M_{x, \mu}^{\dagger}\right)}, \frac{M_{x, \mu} \rho_{k}^{e} M_{x, \mu}^{\dagger}}{\operatorname{Tr}\left(M_{x, \mu} \rho_{k}^{e} M_{x, \mu}^{\dagger}\right)}\right) \quad \text { with probability } \quad p_{x, \mu}\left(\rho_{k}\right)
$$

where $p_{x, \mu}\left(\rho_{k}\right)$ is given by (12) and (11). Then the fidelity $\left(F\left(\rho_{k}, \rho_{k}^{e}\right)\right)$ defined in Equation (1) is a $\left(\mathcal{F}_{k}\right)$ submartingale where $\left.\mathcal{F}_{k}=\sigma\left\{\left(\rho_{l}, \rho_{l}^{e}\right) \mid l \leq k\right)\right\}$. In particular, we have

$$
\mathbb{E}\left(F\left(\rho_{l}, \rho_{l}^{e}\right) \mid \mathcal{F}_{k}\right)=\mathbb{E}\left(F\left(\rho_{l}, \rho_{l}^{e}\right) \mid\left(\rho_{k}, \rho_{k}^{e}\right)\right) \geq F\left(\rho_{k}, \rho_{k}^{e}\right),
$$

for all $l>k$. 


\subsection{Stability of continuous-time filters}

From the description of the quantum trajectory $\left(\rho_{k}\right)$ and its associated quantum filter $\left(\rho_{k}^{e}\right)$ with the time parameter $n$, we can define the associated continuous-time stochastic processes denoted by $\left(\rho_{n}(t)\right)$ and $\left(\rho_{n}^{e}(t)\right)$ with

$$
\rho_{n}(t)=\rho_{[n t]}, \quad \rho_{n}^{e}(t)=\rho_{[n t]}^{e} .
$$

It is clear that $\left(\rho_{n}, \rho_{n}^{e}\right)$ is a Markov process as $\left(\rho_{k}, \rho_{k}^{e}\right)$ is a Markov chain.

Before announcing the main result of this section, first let us show the following proposition.

Proposition 1. The Markov process $\left(\rho_{n}(t), \rho_{n}^{e}(t)\right)$ with $\rho_{n}$ and $\rho_{n}^{e}$ satisfying respectively Equations (13) and (15) converges in distribution, when $n$ goes to infinity, to the Markov process $\left(\rho_{t}, \rho_{t}^{e}\right)$ with $\rho_{t}$ and $\rho_{t}^{e}$ satisfying respectively Equations (2) and (5).

Proof. The approach to prove this proposition is usual in probability theory: we show the convergence of the generator associated to the Markov process $\left(\rho_{n}, \rho_{n}^{e}\right)$ towards the one associated to the Markov process $\left(\rho_{t}, \rho_{t}^{e}\right)$. Also, we need to prove the tightness property of the sequence $\left(\rho_{n}, \rho_{n}^{e}\right)$.

The tightness of the sequence $\left(\rho_{n}(t), \rho_{n}^{e}(t)\right)$ is guaranteed if, for any $T>0$, exists $M>0$ such that for all $0 \leq t_{1} \leq t_{2} \leq T$ and $\forall t \in\left[t_{1}, t_{2}\right]$,

$$
\mathbb{E}\left[\left\|\left(\rho_{n}\left(t_{2}\right), \rho_{n}^{e}\left(t_{2}\right)\right)-\left(\rho_{n}(t), \rho_{n}^{e}(t)\right)\right\|^{2}\left\|\left(\rho_{n}(t), \rho_{n}^{e}(t)\right)-\left(\rho_{n}\left(t_{1}\right), \rho_{n}^{e}\left(t_{1}\right)\right)\right\|^{2}\right] \leq M\left(t_{2}-t_{1}\right)^{2} .
$$

We do not develop the arguments for showing the tightness property. They can be obtained directly with the same arguments as [19].

Thus here, we just focus on the convergence of the generators. Take $\mathcal{A}_{n}$ and $\mathcal{A}$ as the generators associated respectively to the sequences $\left(\rho_{n}, \rho_{n}^{e}\right)$ and $\left(\rho, \rho^{e}\right)$. We have to prove that, for any $C^{2}$-real valued function $\left(\rho, \rho^{e}\right) \mapsto f\left(\rho, \rho^{e}\right)$,

$$
\lim _{n \rightarrow+\infty} \sup _{\left(\rho, \rho^{e}\right) \in \mathcal{D}^{2}}\left|\mathcal{A}_{n} f\left(\rho, \rho^{e}\right)-\mathcal{A} f\left(\rho, \rho^{e}\right)\right|=0 .
$$

We have the following expression for the generator $\mathcal{A} f\left(\rho, \rho^{e}\right)$

$$
\begin{aligned}
& \mathcal{A} f\left(\rho, \rho^{e}\right)=D_{\left(\rho, \rho^{e}\right)} f .\left(-i[H, \rho]+\sum_{\xi} \mathcal{L}_{\xi}(\rho),-i\left[H, \rho^{e}\right]+\sum_{\xi} \mathcal{L}_{\xi}\left(\rho^{e}\right)+K\left(\rho, \rho^{e}\right)\right) \\
&+\frac{1}{2} \sum_{\nu} D_{\left(\rho, \rho^{e}\right)}^{2} f .\left(\Lambda_{\nu}(\rho), \Lambda_{\nu}\left(\rho^{e}\right) ; \Lambda_{\nu}(\rho), \Lambda_{\nu}\left(\rho^{e}\right)\right) \\
&+\sum_{\mu} \operatorname{Tr}\left(V_{\mu} \rho V_{\mu}^{\dagger}\right)\left(f\left(\frac{V_{\mu} \rho V_{\mu}^{\dagger}}{\operatorname{Tr}\left(V_{\mu} \rho V_{\mu}^{\dagger}\right)}, \frac{V_{\mu} \rho^{e} V_{\mu}^{\dagger}}{\operatorname{Tr}\left(V_{\mu} \rho^{e} V_{\mu}^{\dagger}\right)}\right)-f\left(\rho, \rho^{e}\right)-D_{\left(\rho, \rho^{e}\right)} f .\left(\Upsilon_{\mu}(\rho), \Upsilon_{\mu}\left(\rho^{e}\right)\right)\right),
\end{aligned}
$$

where

$$
K\left(\rho, \rho^{e}\right):=\sum_{\mu} \Upsilon_{\mu}\left(\rho^{e}\right)\left(\operatorname{Tr}\left(V_{\mu} \rho V_{\mu}^{\dagger}\right)-\operatorname{Tr}\left(V_{\mu} \rho^{e} V_{\mu}^{\dagger}\right)\right)+\sum_{\nu} \Lambda_{\nu}\left(\rho^{e}\right)\left(\operatorname{Tr}\left(V_{\nu} \rho V_{\nu}^{\dagger}\right)-\operatorname{Tr}\left(V_{\nu} \rho^{e} V_{\nu}^{\dagger}\right)\right)
$$


Now let us calculate the expression of $\mathcal{A}_{n}$. We have, up to $\mathcal{O}\left(n^{-1 / 2}\right)$ terms,

$$
\begin{aligned}
\mathcal{A}_{n} f\left(\rho, \rho^{e}\right) \approx \sum_{x} n p_{x, 0}(\rho) & \left(f\left(\frac{M_{x, 0} \rho M_{x, 0}^{\dagger}}{\operatorname{Tr}\left(M_{x, 0} \rho M_{x, 0}^{\dagger}\right)}, \frac{M_{x, 0} \rho^{e} M_{x, 0}^{\dagger}}{\operatorname{Tr}\left(M_{x, 0} \rho^{e} M_{x, 0}^{\dagger}\right)}\right)-f\left(\rho, \rho^{e}\right)\right) \\
+ & \sum_{x, \mu} n p_{x, \mu}(\rho)\left(f\left(\frac{M_{x, \mu} \rho M_{x, \mu}^{\dagger}}{\operatorname{Tr}\left(M_{x, \mu} \rho M_{x, \mu}^{\dagger}\right)}, \frac{M_{x, \mu} \rho^{e} M_{x, \mu}^{\dagger}}{\operatorname{Tr}\left(M_{x, \mu} \rho^{e} M_{x, \mu}^{\dagger}\right)}\right)-f\left(\rho, \rho^{e}\right)\right) .
\end{aligned}
$$

Since $\frac{M_{x, 0} \rho M_{x, 0}^{\dagger}}{p_{x, 0}(\rho)}=\rho+\mathcal{O}\left(n^{-1 / 2}\right)$ and also, by using Taylor's formula, we have

$$
\begin{gathered}
f\left(\frac{M_{x, 0} \rho M_{x, 0}^{\dagger}}{p_{x, 0}(\rho)}, \frac{M_{x, 0} \rho^{e} M_{x, 0}^{\dagger}}{p_{x, 0}\left(\rho^{e}\right)}\right)-f\left(\rho, \rho^{e}\right)=D_{\left(\rho, \rho^{e}\right)} f \cdot\left(\frac{M_{x, 0} \rho M_{x, 0}^{\dagger}}{p_{x, 0}(\rho)}-\rho, \frac{M_{x, 0} \rho^{e} M_{x, 0}^{\dagger}}{p_{x, 0}\left(\rho^{e}\right)}-\rho^{e}\right) \\
+\frac{1}{2} D_{\left(\rho, \rho^{e}\right)}^{2} f \cdot\left(\frac{M_{x, 0} \rho M_{x, 0}^{\dagger}}{p_{x, 0}(\rho)}-\rho, \frac{M_{x, 0} \rho^{e} M_{x, 0}^{\dagger}}{p_{x, 0}\left(\rho^{e}\right)}-\rho^{e} ; \frac{M_{x, 0} \rho M_{x, 0}^{\dagger}}{p_{x, 0}(\rho)}-\rho, \frac{M_{x, 0} \rho^{e} M_{x, 0}^{\dagger}}{p_{x, 0}\left(\rho^{e}\right)}-\rho^{e}\right)+\mathcal{O}\left(n^{-3 / 2}\right) .
\end{gathered}
$$

Consequently,

$$
\begin{aligned}
& n \sum_{x} p_{x, 0}(\rho)\left(f\left(\frac{M_{x, 0} \rho M_{x, 0}^{\dagger}}{p_{x, 0}(\rho)}, \frac{M_{x, 0} \rho^{e} M_{x, 0}^{\dagger}}{p_{x, 0}\left(\rho^{e}\right)}\right)-f\left(\rho, \rho^{e}\right)\right) \\
& =n D_{\left(\rho, \rho^{e}\right)} f \cdot\left(\sum_{x} M_{x, 0} \rho M_{x, 0}^{\dagger}-p_{x, 0}(\rho) \rho, \sum_{x}\left(M_{x, 0} \rho^{e} M_{x, 0}^{\dagger}-p_{x, 0}\left(\rho^{e}\right) \rho^{e}\right) \frac{p_{x, 0(\rho)}}{p_{x, 0\left(\rho^{e}\right)}}\right) \\
& +\frac{1}{2} \sum_{x} n p_{x, 0}(\rho) D_{\left(\rho, \rho^{e}\right)}^{2} f \cdot\left(\frac{M_{x, 0} \rho M_{x, 0}^{\dagger}}{p_{x, 0}(\rho)}-\rho, \frac{M_{x, 0} \rho^{e} M_{x, 0}^{\dagger}}{p_{x, 0}\left(\rho^{e}\right)}-\rho^{e} ; \frac{M_{x, 0} \rho M_{x, 0}^{\dagger}}{p_{x, 0}(\rho)}-\rho, \frac{M_{x, 0} \rho^{e} M_{x, 0}^{\dagger}}{p_{x, 0}\left(\rho^{e}\right)}-\rho^{e}\right)+\mathcal{O}\left(n^{-1 / 2}\right),
\end{aligned}
$$

where according to $(10)$ and (11), up to $\mathcal{O}\left(n^{-1 / 2}\right)$ terms, we have

$$
\begin{aligned}
n \sum_{x}\left(M_{x, 0} \rho M_{x, 0}^{\dagger}-p_{x, 0}(\rho) \rho\right) & \approx-i[H, \rho]+\sum_{\mu}\left(\operatorname{Tr}\left(\rho V_{\mu}^{\dagger} V_{\mu}\right) \rho-\frac{\left\{\rho, V_{\mu}^{\dagger} V_{\mu}\right\}}{2}\right)+\sum_{\nu} \mathcal{L}_{\nu}(\rho) \\
& =-i[H, \rho]+\sum_{\xi} \mathcal{L}_{\xi}(\rho)-\sum_{\mu} \Upsilon_{\mu}(\rho) \operatorname{Tr}\left(V_{\mu} \rho V_{\mu}^{\dagger}\right)
\end{aligned}
$$

since, for any $\nu, \sum_{x} x_{\nu-m_{P}} \Lambda_{\nu}(\rho)=0$ and $\sum_{x} c_{W}=1$. Also, we have

$$
\begin{aligned}
& M_{x, 0} \rho^{e} M_{x, 0}^{\dagger}-p_{x, 0}\left(\rho^{e}\right) \rho^{e} \\
= & c_{W}\left(\frac{1}{\sqrt{n}} \sum_{\nu} x_{\nu-m_{P}} \Lambda_{\nu}\left(\rho^{e}\right)+\frac{1}{n}\left(-i\left[H, \rho^{e}\right]+\sum_{\xi} \mathcal{L}_{\xi}\left(\rho^{e}\right)-\sum_{\mu} \Upsilon_{\mu}\left(\rho^{e}\right) \operatorname{Tr}\left(V_{\mu} \rho^{e} V_{\mu}^{\dagger}\right)\right)\right) .
\end{aligned}
$$

Therefore, we find

$$
\begin{aligned}
n \sum_{x}\left(M_{x, 0} \rho^{e} M_{x, 0}^{\dagger}\right. & \left.-p_{x, 0}\left(\rho^{e}\right) \rho^{e}\right) \frac{p_{x, 0}(\rho)}{p_{x, 0}\left(\rho^{e}\right)} \\
=-i\left[H, \rho^{e}\right] & +\sum_{\xi} \mathcal{L}_{\xi}\left(\rho^{e}\right)-\sum_{\mu} \Upsilon_{\mu}\left(\rho^{e}\right) \operatorname{Tr}\left(V_{\mu} \rho^{e} V_{\mu}^{\dagger}\right) \\
& +\sum_{\nu} \Lambda_{\nu}\left(\rho^{e}\right)\left(\operatorname{Tr}\left(V_{\nu} \rho V_{\nu}^{\dagger}\right)-\operatorname{Tr}\left(V_{\nu} \rho^{e} V_{\nu}^{\dagger}\right)\right)+\mathcal{O}\left(n^{-\frac{1}{2}}\right)
\end{aligned}
$$


since $\frac{p_{x, 0}(\rho)}{p_{x, 0}\left(\rho^{e}\right)}=1+\frac{1}{\sqrt{n}} \sum_{\nu} x_{\nu-m_{P}}\left(\operatorname{Tr}\left(\left(V_{\nu}+V_{\nu}^{\dagger}\right) \rho\right)-\operatorname{Tr}\left(\left(V_{\nu}+V_{\nu}^{\dagger}\right) \rho^{e}\right)\right)+\mathcal{O}\left(n^{-1 / 2}\right)$. Moreover, we have used the fact that

$$
\begin{aligned}
\sum_{x} \sum_{\nu} c_{W} x_{\nu-m_{P}} \Lambda_{\nu}\left(\rho^{e}\right) \sum_{\nu^{\prime}} x_{\nu^{\prime}-m_{P}}\left(\operatorname{Tr}\left(\left(V_{\nu^{\prime}}+V_{\nu^{\prime}}^{\dagger}\right) \rho\right)-\operatorname{Tr}\left(\left(V_{\nu^{\prime}}+V_{\nu^{\prime}}^{\dagger}\right) \rho^{e}\right)\right) \\
=\sum_{\nu} \Lambda_{\nu}\left(\rho^{e}\right)\left(\operatorname{Tr}\left(\left(V_{\nu}+V_{\nu}^{\dagger}\right) \rho\right)-\operatorname{Tr}\left(\left(V_{\nu}+V_{\nu}^{\dagger}\right) \rho^{e}\right)\right),
\end{aligned}
$$

since $x_{\nu-m_{P}}^{2}=1, \sum_{x} c_{W} x_{\nu-m_{P}}^{2}=1$ and

$$
\forall \nu \neq \nu^{\prime}: \quad \sum_{x} c_{W} x_{\nu-m_{P}} x_{\nu^{\prime}-m_{P}} \Lambda_{\nu}\left(\rho^{e}\right)\left(\operatorname{Tr}\left(\left(V_{\nu^{\prime}}+V_{\nu^{\prime}}^{\dagger}\right) \rho\right)-\operatorname{Tr}\left(\left(V_{\nu^{\prime}}+V_{\nu^{\prime}}^{\dagger}\right) \rho^{e}\right)\right)=0 .
$$

Equation (19) can be rewritten as follows

$$
\begin{aligned}
n \sum_{x}\left(M_{x, 0} \rho^{e} M_{x, 0}^{\dagger}-p_{x, 0}\left(\rho^{e}\right) \rho^{e}\right) \frac{p_{x, 0}(\rho)}{p_{x, 0}\left(\rho^{e}\right)} & \\
& =-i\left[H, \rho^{e}\right]+\sum_{\xi} \mathcal{L}_{\xi}\left(\rho^{e}\right)+K\left(\rho, \rho^{e}\right)-\sum_{\mu} \Upsilon_{\mu}\left(\rho^{e}\right) \operatorname{Tr}\left(V_{\mu} \rho V_{\mu}^{\dagger}\right)+\mathcal{O}\left(n^{-\frac{1}{2}}\right) .
\end{aligned}
$$

As a result, the first righthand side term in Equation (17) can be written as follows

$$
\begin{aligned}
n D_{\left(\rho, \rho^{e}\right)} f & \left(\sum_{x} M_{x, 0} \rho M_{x, 0}^{\dagger}-p_{x, 0}(\rho) \rho, \sum_{x}\left(M_{x, 0} \rho^{e} M_{x, 0}^{\dagger}-p_{x, 0}\left(\rho^{e}\right) \rho^{e}\right) \frac{p_{x, 0(\rho)}}{p_{x, 0\left(\rho^{e}\right)}}\right) \\
=D_{\left(\rho, \rho^{e}\right)} f \cdot(-i[H, \rho] & \left.+\sum_{\xi} \mathcal{L}_{\xi}(\rho),-i\left[H, \rho^{e}\right]+\sum_{\xi} \mathcal{L}_{\xi}\left(\rho^{e}\right)+K\left(\rho, \rho^{e}\right)\right) \\
& -\sum_{\mu} \operatorname{Tr}\left(V_{\mu} \rho V_{\mu}^{\dagger}\right) D_{\left(\rho, \rho^{e}\right)} f \cdot\left(\Upsilon_{\mu}(\rho), \Upsilon_{\mu}\left(\rho^{e}\right)\right)+\mathcal{O}\left(n^{-1 / 2}\right) .
\end{aligned}
$$

Now let us calculate the second righthand side term in Equation (17). To get the zero order terms of $\sum_{x} n p_{x, 0}(\rho) D_{\left(\rho, \rho^{e}\right)}^{2} f \cdot\left(\frac{M_{x, 0} \rho M_{x, 0}^{\dagger}}{p_{x, 0}(\rho)}-\rho, \frac{M_{x, 0} \rho^{e} M_{x, 0}^{\dagger}}{p_{x, 0}\left(\rho^{e}\right)}-\rho^{e} ; \frac{M_{x, 0} \rho M_{x, 0}^{\dagger}}{p_{x, 0}(\rho)}-\rho, \frac{M_{x, 0} \rho^{e} M_{x, 0}^{\dagger}}{p_{x, 0}\left(\rho^{e}\right)}-\rho^{e}\right)$, we just need to combine the terms of order $n^{-1 / 2}$ in $\frac{M_{x, 0} \rho M_{x, 0}^{\dagger}}{p_{x, 0}(\rho)}-\rho$ and in $M_{x, 0} \rho M_{x, 0}^{\dagger}-p_{x, 0}(\rho) \rho$. Since

$$
M_{x, 0} \rho M_{x, 0}^{\dagger}-p_{x, 0}(\rho) \rho=\frac{c_{W}}{\sqrt{n}}\left(\sum_{\nu} x_{\nu-m_{P}} \Lambda_{\nu}(\rho)\right)+\mathcal{O}\left(n^{-1 / 2}\right)
$$

and

$$
\frac{M_{x, 0} \rho M_{x, 0}^{\dagger}}{p_{x, 0}(\rho)}-\rho \approx \frac{\rho+\frac{1}{\sqrt{n}} \sum_{\nu} x_{\nu-m_{P}}\left(V_{\nu} \rho+\rho V_{\nu}^{\dagger}\right)}{1+\frac{1}{\sqrt{n}} \sum_{\nu} x_{\nu-m_{P}} \operatorname{Tr}\left(\left(V_{\nu}+V_{\nu}^{\dagger}\right) \rho\right)}-\rho \approx \frac{1}{\sqrt{n}}\left(\sum_{\nu} x_{\nu-m_{P}} \Lambda_{\nu}(\rho)\right)+\mathcal{O}\left(n^{-1 / 2}\right),
$$

we get, up to $\mathcal{O}\left(n^{-1 / 2}\right)$ terms

$$
\begin{gathered}
\sum_{x} n p_{x, 0}(\rho) D_{\left(\rho, \rho^{e}\right)}^{2} f \cdot\left(\frac{M_{x, 0} \rho M_{x, 0}^{\dagger}}{p_{x, 0}(\rho)}-\rho, \frac{M_{x, 0} \rho^{e} M_{x, 0}^{\dagger}}{p_{x, 0}\left(\rho^{e}\right)}-\rho^{e} ; \frac{M_{x, 0} \rho M_{x, 0}^{\dagger}}{p_{x, 0}(\rho)}-\rho, \frac{M_{x, 0} \rho^{e} M_{x, 0}^{\dagger}}{p_{x, 0}\left(\rho^{e}\right)}-\rho^{e}\right) \approx \\
\sum_{x, \nu, \nu^{\prime}} c_{W} x_{\nu-m_{P}} x_{\nu^{\prime}} D_{\left(\rho, \rho^{e}\right)}^{2} f \cdot\left(\Lambda_{\nu}(\rho), \Lambda_{\nu}\left(\rho^{e}\right) ; \Lambda_{\nu^{\prime}}(\rho), \Lambda_{\nu^{\prime}}\left(\rho^{e}\right)\right)=\sum_{\nu} D_{\left(\rho, \rho^{e}\right)}^{2} f \cdot\left(\Lambda_{\nu}(\rho), \Lambda_{\nu}\left(\rho^{e}\right) ; \Lambda_{\nu}(\rho), \Lambda_{\nu}\left(\rho^{e}\right)\right),
\end{gathered}
$$


where for the above equality, we have used the following facts $x_{\nu-m_{P}}^{2}=1, \quad \sum_{x} c_{W}=1 \quad$ and $\quad \forall \nu \neq \nu^{\prime}: \quad \sum_{x} c_{W} x_{\nu-m_{P}} x_{\nu^{\prime}-m_{P}} D_{\rho}^{2} f(\rho) \cdot\left(\Lambda_{\nu}(\rho), \Lambda_{\nu^{\prime}}(\rho)\right)=0$.

Thus, we find

$$
\begin{aligned}
\sum_{x} p_{x, 0}(\rho)\left(f\left(\frac{M_{x, 0} \rho M_{x, 0}^{\dagger}}{p_{x, 0}(\rho)}, \frac{M_{x, 0} \rho^{e} M_{x, 0}^{\dagger}}{p_{x, 0}\left(\rho^{e}\right)}\right)-f\left(\rho, \rho^{e}\right)\right) \\
=D_{\left(\rho, \rho^{e}\right)} f \cdot\left(-i[H, \rho]+\sum_{\xi} \mathcal{L}_{\xi}(\rho),-i\left[H, \rho^{e}\right]+\sum_{\xi} \mathcal{L}_{\xi}\left(\rho^{e}\right)+K\left(\rho, \rho^{e}\right)\right) \\
\quad-\sum_{\mu} \operatorname{Tr}\left(V_{\mu} \rho V_{\mu}^{\dagger}\right) D_{\left(\rho, \rho^{e}\right)} f \cdot\left(\Upsilon_{\mu}(\rho), \Upsilon_{\mu}\left(\rho^{e}\right)\right) . \\
+\frac{1}{2} \sum_{\nu} D_{\rho}^{2} f(\rho) \cdot\left(\Lambda_{\nu}(\rho), \Lambda_{\nu}\left(\rho^{e}\right) ; \Lambda_{\nu}(\rho), \Lambda_{\nu}\left(\rho^{e}\right)\right)+\mathcal{O}\left(n^{-1 / 2}\right) .
\end{aligned}
$$

According to (12), we have also

$$
\begin{aligned}
\sum_{x, \mu} n p_{x, \mu}(\rho)\left(f\left(\frac{M_{x, \mu} \rho M_{x, \mu}^{\dagger}}{\operatorname{Tr}\left(M_{x, \mu} \rho M_{x, \mu}^{\dagger}\right)}, \frac{M_{x, \mu} \rho^{e} M_{x, \mu}^{\dagger}}{\operatorname{Tr}\left(M_{x, \mu} \rho^{e} M_{x, \mu}^{\dagger}\right)}\right)-f\left(\rho, \rho^{e}\right)\right) \\
=\sum_{\mu} \operatorname{Tr}\left(V_{\mu} \rho V_{\mu}^{\dagger}\right)\left(f\left(\frac{V_{\mu} \rho V_{\mu}^{\dagger}}{\operatorname{Tr}\left(V_{\mu} \rho V_{\mu}^{\dagger}\right)}, \frac{V_{\mu} \rho^{e} V_{\mu}^{\dagger}}{\operatorname{Tr}\left(V_{\mu} \rho^{e} V_{\mu}^{\dagger}\right)}\right)-f\left(\rho, \rho^{e}\right)\right) .
\end{aligned}
$$

Finally, by Equations (20) and (21), we find that

$$
\mathcal{A}_{n} f\left(\rho, \rho^{e}\right)=\mathcal{A} f\left(\rho, \rho^{e}\right)+\mathcal{O}\left(n^{-1 / 2}\right),
$$

which finishes the proof of Proposition 1.

Now we are in the state to announce the main result of this section.

Theorem 2. Consider the Markov process $\left(\rho_{t}, \rho_{t}^{e}\right)$ satisfying respectively Equations (2) and (5). Then the fidelity $\left(F\left(\rho_{t}, \rho_{t}^{e}\right)\right)$ defined in Equation (1) is a $\left(\mathcal{F}_{t}\right)$-submartingale, where $\mathcal{F}_{t}=\sigma\left\{\left(\rho_{\tau}, \rho_{\tau}^{e}\right) \mid \tau \leq t\right\}$. In particular, we have,

$$
\mathbb{E}\left(F\left(\rho_{\tau}, \rho_{\tau}^{e}\right) \mid \mathcal{F}_{t}\right)=\mathbb{E}\left(F\left(\rho_{\tau}, \rho_{\tau}^{e}\right) \mid\left(\rho_{t}, \rho_{t}^{e}\right)\right) \geq F\left(\rho_{t}, \rho_{t}^{e}\right),
$$

for all $\tau \geq t$.

Proof. By Theorem 1, we know that the fidelity $F\left(\rho_{n}, \rho_{n}^{e}\right)$ is a submartingale with respect to the natural filtration of $\left(\rho_{k}, \rho_{k}^{e}\right)$. In terms of $\left(\rho_{n}(t), \rho_{n}^{e}(t)\right.$, it follows that

$$
\mathbb{E}\left(F\left(\rho_{n}(\tau), \rho_{n}^{e}(\tau)\right) \mid\left(\rho_{n}(t), \rho_{n}^{e}(t)\right)\right) \geq F\left(\rho_{n}(t), \rho_{n}^{e}(t)\right),
$$

for all $\tau \geq t$. In Proposition 1, we showed that $\left(\rho_{n}(t), \rho_{n}^{e}(t)\right)$ converges in distribution to $\left(\rho_{t}, \rho_{t}^{e}\right)$. This implies that for any continuous real function $f\left(\rho, \rho^{e}\right)$ we have

$$
\forall \tau \geq t, \quad \lim _{n \rightarrow+\infty} \mathbb{E}\left(f\left(\rho_{n}(\tau), \rho_{n}^{e}(\tau)\right) \mid\left(\rho_{n}(t), \rho_{n}^{e}(t)\right)=\mathbb{E}\left(f\left(\rho_{\tau}, \rho_{\tau}^{e}\right) \mid\left(\rho_{t}, \rho_{t}^{e}\right)\right) .\right.
$$

As $F$ is continuous, the limit of (22) for $n$ tending to $\infty$ yields $\mathbb{E}\left(F\left(\rho_{\tau}, \rho_{\tau}^{e}\right) \mid\left(\rho_{t}, \rho_{t}^{e}\right)\right) \geq F\left(\rho_{t}, \rho_{t}^{e}\right)$ for all $\tau \geq t$. Since $\left(\rho_{t}, \rho_{t}^{e}\right)$ is a Markov process, the result follows. 


\section{Imperfect measurements}

By imperfect measurements, we mean both unread measurements performed by the environment (decoherence) and active measurements performed by non-ideal detectors. Starting from the discrete-time case experimentally used in [22] and detailed in [23], we derive the continuous-time optimal filters driven either by Poisson, Wiener processes or both of them. We obtain the SMEs and their associated quantum filters.

\subsection{Discrete-time filters}

The presentation here is very much inspired from [23] and adapted to (13) for $n$ large. The jump-events labelled by $(x, z)$ are of type $(x, \mu)$ with $\mu \in\left\{0,1, \cdots, m_{P}\right\}$. The effectively measured events are labelled by $s \in\{1, \ldots, m\}$, with $m$ denoting the number of distinct experimental detector outcomes. Suppose that we know the correlation between the jumpevents $(x, \mu)$ and the experimental detection $s$. These correlations are modeled here by classical probabilities through a stochastic matrix $\eta: \eta_{s,(x, \mu)}^{n}$ that gives the probability of experimental detection $s$ knowing that the effective jump-event is $(x, \mu)$. Since $\eta_{s,(x, \mu)}^{n} \geq 0$ and for each $(x, \mu), \sum_{s=1}^{m} \eta_{s,(x, \mu)}^{n}=1$, the matrix $\eta^{n}=\left(\eta_{s,(x, \mu)}^{n}\right)$ is a left stochastic matrix. We assume the following asymptotic for $\eta^{n}$,

$$
\eta^{n}=\eta^{\infty}+\frac{\widetilde{\eta}}{n}+\mathcal{O}\left(n^{-2}\right)
$$

where $\eta^{\infty}$ is a left stochastic matrix.

We still denote by $\rho$ the state associated to these experimental detections which is the best estimation of the system state knowing the initial state and all previous experimental detections. Following [23], it obeys to the following Markov process:

$$
\rho_{k+1}=\frac{\mathcal{M}_{s}^{n}\left(\rho_{k}\right)}{\operatorname{Tr}\left(\mathcal{M}_{s}^{n}\left(\rho_{k}\right)\right)} \quad \text { with probability } p_{s}\left(\rho_{k}\right)=\operatorname{Tr}\left(\mathcal{M}_{s}^{n}\left(\rho_{k}\right)\right)
$$

where $\mathcal{M}_{s}^{n}(\rho) \triangleq \sum_{x, \mu} \eta_{s,(x, \mu)}^{n} M_{x, \mu} \rho M_{x, \mu}^{\dagger}, M_{x, 0}$ and $M_{x, \mu}$ are given respectively by Equations (7) and (8) depending also on $n$.

Suppose that the initial state of dynamics (24) is not well known. Let $\rho_{0}^{e}$ be an arbitrary initial estimate, the estimate discrete-time filter satisfies the following dynamics

$$
\rho_{k+1}^{e}=\frac{\mathcal{M}_{s_{k}}^{n}\left(\rho_{k}^{e}\right)}{\operatorname{Tr}\left(\mathcal{M}_{s_{k}}^{n}\left(\rho_{k}^{e}\right)\right)}
$$

where $s_{k}$ corresponds to the experimental detection at time-step $k$.

Remark 2. For the same reason given in Remark 1, the above description is not always valid. Since the normalization $\operatorname{Tr}\left(\mathcal{M}_{s}^{n}\left(\rho_{k}^{e}\right)\right)$ can vanish and the formula $(25)$ is then not well defined. Such a problem will not be appeared when $\rho_{k}^{e}$ is full rank. (When $\operatorname{Tr}\left(\mathcal{M}_{s}^{n}\left(\rho_{k}^{e}\right)\right)=0$, we can still define the value of $\rho_{k+1}^{e}$, see more details in [23].) Again, as we consider the asymptotic evolution, such a problem will not appear.

We now state a theorem ensuring the stability of such estimation procedure whatever the initial state $\rho_{0}^{e}$ is. 
Theorem 3 ([21, 23]). Consider the Markov chain $\left(\rho_{k}, \rho_{k}^{e}\right)$ satisfying $(24)$ and $(25)$ :

$$
\left(\rho_{k+1}, \rho_{k+1}^{e}\right)=\left(\frac{\mathcal{M}_{s}^{n}\left(\rho_{k}\right)}{\operatorname{Tr}\left(\mathcal{M}_{s}^{n}\left(\rho_{k}\right)\right)}, \frac{\mathcal{M}_{s}^{n}\left(\rho_{k}^{e}\right)}{\operatorname{Tr}\left(\mathcal{M}_{s}^{n}\left(\rho_{k}^{e}\right)\right)}\right) \quad \text { with probability } p_{s}\left(\rho_{k}\right)=\operatorname{Tr}\left(\mathcal{M}_{s}^{n}\left(\rho_{k}\right)\right) .
$$

Then the fidelity $\left(F\left(\rho_{k}, \rho_{k}^{e}\right)\right)$ is a $\left(\mathcal{F}_{k}\right)$-submartingale where $\left.\mathcal{F}_{k}=\sigma\left\{\left(\rho_{l}, \rho_{l}^{e}\right) \mid l \leq k\right)\right\}$. In particular, we have

$$
\mathbb{E}\left(F\left(\rho_{l}, \rho_{l}^{e}\right) \mid \mathcal{F}_{k}\right)=\mathbb{E}\left(F\left(\rho_{l}, \rho_{l}^{e}\right) \mid\left(\rho_{k}, \rho_{k}^{e}\right)\right) \geq F\left(\rho_{k}, \rho_{k}^{e}\right),
$$

for all $l>k$.

As far as we know, the continuous-time asymptotic versions of the discrete-time dynamics (24) and (25) associated to a left stochastic matrix $\eta^{n}$ with asymptotics (23), have not been established up to now. In the following, we derive such continuous-time SMEs which are the limits of these discrete-time dynamics and prove their stability.

\subsection{Continuous-time filters as limit of discrete-time filters}

Take $n$ large and consider the piece-wise constant continuous-time stochastic processes denoted by $\rho_{n}(t)$ and $\rho_{n}^{e}(t)$ with

$$
\rho_{n}(t)=\rho_{[n t]}, \quad \rho_{n}^{e}(t)=\rho_{[n t]}^{e} .
$$

where the discrete-time process $\left(\rho_{k}, \rho_{k}^{e}\right)$ obeys to $(24)$ with $k=[n t]$, the entire part of $n t$. It is clear that $\left(\rho_{n}(t), \rho_{n}^{e}(t)\right)$ is a Markov process, as $\left(\rho_{k}, \rho_{k}^{e}\right)$ is the Markov chain of Theorem 3.

Now suppose that $\rho_{t}$ and $\rho_{t}^{e}$ be respectively the solutions of the continuous-time dynamics of the true filter and its estimate at time $t$. Let $\mathcal{A}_{n}$ and $\mathcal{A}$ be respectively the Markov generators of $\left(\rho_{n}, \rho_{n}^{e}\right)$ and $\left(\rho, \rho^{e}\right)$. Then for all $C^{2}$-real valued function $f$, we are looking for the continuous-time processes $\rho$ and $\rho^{e}$ such that the following limit holds.

$$
\lim _{n \rightarrow \infty} \sup _{\left(\rho, \rho^{e}\right) \in \mathcal{D}^{2}}\left|\mathcal{A}_{n} f\left(\rho, \rho^{e}\right)-\mathcal{A} f\left(\rho, \rho^{e}\right)\right|=0 .
$$

We will see that such continuous-time limit depends essentially on the structure of $\eta^{\infty}=$ $\lim _{n \rightarrow \infty} \eta^{n}$ described in Lemma 1 and yields a generalization of the usual stochastic master equations driven by Wiener and Poisson processes.

Lemma 1. Take the left stochastic matrix $\eta_{s,(x, \mu)}^{\infty}$ defined by (23) with line index $s \in$ $\{1, \ldots, m\}$ and column index $(x, \mu) \in\{-1,1\}^{m_{W}} \times\left\{0,1, \ldots, m_{P}\right\}$. Consider the following partition $\left(S^{W}, S^{P}\right)$ of $\{1, \cdots, m\}$ labeling the number of experimental detections:

$S^{P}=\left\{s \in\{1, \ldots, m\} \mid \sum_{x \in\{-1,1\}^{m_{W}}} \eta_{s,(x, 0)}^{\infty}=0\right\}, \quad S^{W}=\left\{s \in\{1, \ldots, m\} \mid \sum_{x \in\{-1,1\}^{m_{W}}} \eta_{s,(x, 0)}^{\infty}>0\right\}$.

Then we have

- The $\bar{\eta}_{s, \mu}$ 's defined by

$$
\bar{\eta}_{s, \mu} \triangleq 2^{-m_{W}} \sum_{x \in\{-1,1\}^{m_{W}}} \eta_{s,(x, \mu)}^{\infty}, \quad(s, \mu) \in S^{P} \times\left\{1, \ldots, m_{P}\right\},
$$

satisfy

$$
\forall(s, \mu) \in S^{P} \times\left\{1, \ldots, m_{P}\right\}, 0 \leq \bar{\eta}_{s, \mu} \leq 1 \text { and } \forall \mu \in\left\{1, \ldots, m_{P}\right\} \sum_{s \in S^{P}} \bar{\eta}_{s, \mu} \leq 1 .
$$


- The singular values of the matrix $E$ with entries

$$
\forall(s, \nu) \in S^{W} \times\left\{m_{P}, \ldots, m_{P}+m_{W}\right\}, E_{s, \nu} \triangleq 2^{-m_{W} / 2} \frac{\sum_{x} x_{\nu-m_{P}} \eta_{s,(x, 0)}^{\infty}}{\sqrt{\sum_{x} \eta_{s,(x, 0)}^{\infty}}}
$$

belong to $[0,1]$.

Proof. Inequality (28) is a direct consequence of $S^{P}$ definition, because for any $(x, \mu), \sum_{s} \eta_{s,(x, \mu)}^{\infty}=$ 1 and $x$ belongs to a set of cardinal $2^{m_{W}}$.

The matrix $E$ is well defined because, by definition of $S^{W}$, the denominators in (29) are all strictly positive. The singular values of $E$ do not exceed 1, if and only if, for any unitary vector $z \in \mathbb{R}^{m_{W}}$, the Euclidian norm of $E z$ does not exceed 1 . With $z=\left(z_{j}\right)$ we have

$$
\|E z\|^{2}=2^{-m_{W}} \sum_{s \in S^{W}} \frac{\left(\sum_{x, j} x_{j} z_{j} \eta_{s,(x, 0)}^{\infty}\right)^{2}}{\sum_{x} \eta_{s,(x, 0)}^{\infty}}
$$

where $(x, j)$ varies in $\{-1,1\}^{m_{W}} \times\left\{1, \ldots, m_{W}\right\}$. With $\vartheta_{s}=\sum_{x} \eta_{s,(x, 0)}^{\infty}, \vartheta_{s, x}=\frac{\eta_{s,(x, 0)}^{\infty}}{\vartheta_{s}}$ and $\langle x \mid z\rangle=\sum_{j} x_{j} z_{j}$ we have $\|E z\|^{2}=2^{-m_{W}} \sum_{s \in S_{W}} \vartheta_{s}\left(\sum_{x}\langle x \mid z\rangle \vartheta_{s, x}\right)^{2}$. By convexity of $\alpha \mapsto \alpha^{2}$, we have

$$
\forall s \in S^{W}, \quad\left(\sum_{x} \vartheta_{s, x}\langle x \mid z\rangle\right)^{2} \leq \sum_{x} \vartheta_{s, x}(\langle x \mid z\rangle)^{2}
$$

since $\sum_{x} \vartheta_{s, x}=1$ and $\vartheta_{s, x} \geq 0$. Thus

$$
\|E z\|^{2} \leq 2^{-m_{W}} \sum_{s \in S^{W}} \eta_{s,(x, 0)}^{\infty} \sum_{x}(\langle x \mid z\rangle)^{2}=2^{-m_{W}} \sum_{x}(\langle x \mid z\rangle)^{2}
$$

since $\sum_{s} \eta_{s,(x, 0)}^{\infty}=1$ for any $x$. We have

$$
\sum_{x}(\langle x \mid z\rangle)^{2}=\sum_{x, j, j^{\prime}} x_{j} x_{j^{\prime}} z_{j} z_{j^{\prime}}=\sum_{j, j^{\prime}} z_{j} z_{j}^{\prime}\left(\sum_{x} x_{j} x_{j^{\prime}}\right)=\sum_{j} z_{j}^{2}\left(\sum_{x} x_{j}^{2}\right)=2^{m_{W}} \sum_{j} z_{j}^{2}
$$

since $x$ in $\{-1,1\}^{m_{W}}$ implies that $\sum_{x} x_{j} x_{j^{\prime}}=0$ for $j \neq j^{\prime}$. Thus $\|E z\|^{2} \leq 1$ when $\|z\|=1$.

Next theorem provides a generalization of usual SME driven by Wiener processes with detection errors to SME driven simultaneously by Wiener and Poisson processes with detections errors.

Theorem 4. Consider $\left(\rho_{n}(t), \rho_{n}^{e}(t)\right)$ defined by $(26)$ and associated to $(24,25)$ with a left stochastic matrix $\eta^{n}$ verifying (23). Then, for $n \rightarrow+\infty$, the process $\left(\rho_{n}(t), \rho_{n}^{e}(t)\right)$ converges in distribution to the unique solutions of

$$
\begin{aligned}
& d \rho_{t}=-i\left[H, \rho_{t}\right] d t+\left(\sum_{\xi} V_{\xi} \rho_{t} V_{\xi}^{\dagger}-\frac{1}{2}\left(V_{\xi}^{\dagger} V_{\xi} \rho_{t}+\rho_{t} V_{\xi}^{\dagger} V_{\xi}\right)\right) d t \\
& +\sum_{s \in S^{P}}\left(\frac{\bar{\theta}_{s} \rho_{t}+\sum_{\mu} \bar{\eta}_{s, \mu} V_{\mu} \rho_{t} V_{\mu}^{\dagger}}{\bar{\theta}_{s}+\sum_{\mu} \bar{\eta}_{s, \mu} \operatorname{Tr}\left(V_{\mu} \rho_{t} V_{\mu}^{\dagger}\right)}-\rho_{t}\right)\left(d N_{s}(t)-\left(\bar{\theta}_{s}+\sum_{\mu} \bar{\eta}_{s, \mu} \operatorname{Tr}\left(V_{\mu} \rho_{t} V_{\mu}^{\dagger}\right)\right) d t\right) \\
& +\sum_{s \in S^{W}} \sqrt{\bar{\eta}_{s}}\left(\sum_{\nu} c_{s, \nu}\left(V_{\nu} \rho_{t}+\rho_{t} V_{\nu}^{\dagger}-\operatorname{Tr}\left(\left(V_{\nu}+V_{\nu}^{\dagger}\right) \rho_{t}\right) \rho_{t}\right)\right) d W_{s}(t)
\end{aligned}
$$


and

$$
\begin{gathered}
d \rho_{t}^{e}=-i\left[H, \rho_{t}^{e}\right]+\left(\sum_{\xi} V_{\xi} \rho_{t}^{e} V_{\xi}^{\dagger}-\frac{1}{2}\left(V_{\xi}^{\dagger} V_{\xi} \rho_{t}^{e}+\rho_{t}^{e} V_{\xi}^{\dagger} V_{\xi}\right)\right) d t \\
+\sum_{s \in S^{P}}\left(\frac{\bar{\theta}_{s} \rho_{t}^{e}+\sum_{\mu} \bar{\eta}_{s, \mu} V_{\mu} \rho_{t}^{e} V_{\mu}^{\dagger}}{\bar{\theta}_{s}+\sum_{\mu} \bar{\eta}_{s, \mu} \operatorname{Tr}\left(V_{\mu} \rho_{t}^{e} V_{\mu}^{\dagger}\right)}-\rho_{t}^{e}\right)\left(d N_{s}(t)-\left(\bar{\theta}_{s}+\sum_{\mu} \bar{\eta}_{s, \mu} \operatorname{Tr}\left(V_{\mu} \rho_{t}^{e} V_{\mu}^{\dagger}\right)\right) d t\right) \\
+\sum_{s \in S^{W}} \sqrt{\bar{\eta}_{s}}\left(\sum_{\nu} c_{s, \nu}\left(V_{\nu} \rho_{t}^{e}+\rho_{t}^{e} V_{\nu}^{\dagger}-\operatorname{Tr}\left(\left(V_{\nu}+V_{\nu}^{\dagger}\right) \rho_{t}^{e}\right) \rho_{t}^{e}\right)\right) \times \ldots \\
\ldots \times\left(d y_{s}(t)-\sqrt{\bar{\eta}_{s}} \operatorname{Tr}\left(\sum_{\nu} c_{s, \nu}\left(V_{\nu}+V_{\nu}^{\dagger}\right) \rho_{t}^{e}\right) d t .\right)
\end{gathered}
$$

with partition $\left(S_{P}, S_{W}\right)$ of $\{1, \ldots, m\}$ defined in Lemma 1 , with, in the above sums, $\mu \in$ $\left\{1, \ldots, m_{P}\right\}, \nu \in\left\{m_{P}+1, \ldots, m_{P}+m_{W}\right\}$ and $\xi \in\left\{1, \ldots, m_{P}+m_{W}\right\}$, and where

- $s \in S^{P}$ is related to the Poisson process $d N_{s}(t)$ characterized by

$$
\left\langle d N_{s}(t)\right\rangle=\left(\bar{\theta}_{s}+\sum_{\mu} \bar{\eta}_{s, \mu} \operatorname{Tr}\left(V_{\mu} \rho_{t} V_{\mu}^{\dagger}\right)\right) d t
$$

with $\bar{\eta}_{s, \mu}$ given by (27) and $\bar{\theta}_{s}=2^{-m_{W}} \sum_{x} \widetilde{\eta}_{s,(x, 0)} \geq 0(\widetilde{\eta}$ defined in (23)).

- $s \in S^{W}$ is related to the continuous signal $y_{s}$ related to the Wiener process $d W_{s}$ via

$$
d y_{s}(t)=d W_{s}(t)+\sqrt{\bar{\eta}_{s}} \operatorname{Tr}\left(\sum_{\nu} c_{s, \nu}\left(V_{\nu}+V_{\nu}^{\dagger}\right) \rho_{t}\right) d t .
$$

The efficiencies $\bar{\eta}_{s}$ belong to $[0,1]$ and correspond to the eigenvalues of $E E^{\dagger}$ with matrix $E$ defined in Lemma 1. The real coefficients $c_{s, \nu}$ are given by the entries of the orthogonal matrix $C$ appearing in the singular value decomposition of $E=R D C$ with $R$ and $C$ orthogonal and $D$ the rectangular diagonal matrix with diagonal entries $\sqrt{\bar{\eta}_{s}}$ :

$$
\forall s, s^{\prime} \in S^{W}, \sum_{\nu} c_{s, \nu} c_{s^{\prime}, \nu}=\delta_{s, s^{\prime}}
$$

The proof of this theorem is given in the next subsection. It admits the same structure as the proof of Proposition 1 but with sightly more complicated computations for the Markov generators. The stability of such quantum filters is ensured in the following theorem.

Theorem 5. Consider the Markov process $\left(\rho_{t}, \rho_{t}^{e}\right)$, satisfying $(30,31)$ where the positive integers $m_{P}, m_{W}$ and $m$ are arbitrary, where the $m_{p}+m_{W}$ square matrices $V_{\xi}$ are arbitrary, where the partition $\left(S^{W}, S^{P}\right)$ of $\{1, \ldots, m\}$ is arbitrary, where $\forall(s, \mu) \in S^{P} \times\left\{1, \ldots, m_{P}\right\}$, $\bar{\theta}_{s} \geq 0, \bar{\eta}_{s, \mu} \in[0,1]$ and $\sum_{s^{\prime} \in S^{P}} \bar{\eta}_{s^{\prime}, \mu} \leq 1$, where

$$
\forall\left(s, s^{\prime}\right) \in S^{W}, \quad \bar{\eta}_{s} \in[0,1] \quad \text { and } \sum_{\nu \in\left\{m_{P}+1, \ldots, m_{P}+m_{W}\right\}} c_{s, \nu} c_{s^{\prime}, \nu}=\delta_{s, s^{\prime}} .
$$


Then, the fidelity $F\left(\rho_{t}, \rho_{t}^{e}\right)$ defined in Equation $(1)$ is a $\left(\mathcal{F}_{t}\right)$-submartingale, where $\mathcal{F}_{t}=$ $\sigma\left\{\left(\rho_{\tau}, \rho_{\tau}^{e}\right) \mid \tau \leq t\right\}$. In particular, we have,

$$
\mathbb{E}\left(F\left(\rho_{\tau}, \rho_{\tau}^{e}\right) \mid \mathcal{F}_{t}\right)=\mathbb{E}\left(F\left(\rho_{\tau}, \rho_{\tau}^{e}\right) \mid\left(\rho_{t}, \rho_{t}^{e}\right)\right) \geq F\left(\rho_{t}, \rho_{t}^{e}\right),
$$

for all $\tau \geq t$.

Proof. The proof is the similar to the one given for Theorem 2. It relies on a direct application of Theorem 3 and Theorem 4. The assumptions made on the real coefficients $\bar{\theta}_{s}, \bar{\eta}_{s, m u}, \bar{\eta}_{s}$ and $c_{s, \nu}$ implies the existence of a family of stochastic process $\left(\rho_{n}(t), \rho_{n}^{e}(t)\right)$ defined in $(26)$ converging towards $\left(\rho_{t}, \rho^{e}(t)\right)$ for $n$ large. This implication relies on manipulations based on Lemma 1 and providing a family of stochastic matrices $\eta^{n}=\eta^{\infty}+\widetilde{\eta} / n+O(1 / n)$ such that these coefficients $\left(\bar{\theta}_{s}, \bar{\eta}_{s, m u}, \bar{\eta}_{s}, c_{s, \nu}\right)$ are related to $\eta^{\infty}$ and $\widetilde{\eta}$ according to Theorem 4 . These manipulations are simple and not detailed here.

\subsection{Proof of Theorem 4}

The tightness property of $\left(\rho_{n}(t), \rho_{n}^{e}(t)\right)$ can be concluded by the similar argument appeared in [19]. We give here the convergence proof of the Markov generators $\mathcal{A}_{n}$ of $\left(\rho_{n}(t), \rho_{n}^{e}(t)\right)$ towards the Markov generator $\mathcal{A}$ of $\left(\rho_{t}, \rho_{t}^{e}\right)$. We detail the convergence proof for $S^{P}=\varnothing$ with $S^{W}=\{1, \ldots, m\}$ and for $S^{P}=\{1, \ldots, m\}$ with $S^{W}=\varnothing$. The general case where both $S^{P}$ and $S^{W}$ are not empty is just a concatenation of the two previous ones .

\subsubsection{Proof of Theorem 4 when $S^{P}=\varnothing$ and $S^{W}=\{1, \ldots, m\}$}

Consider the singular value decomposition of matrix $E$ defined in Lemma 1: $E=R D C$ with $R \in O(m), C \in O\left(m_{W}\right)$ and $D$ the rectangular diagonal matrix with diagonal formed by $\sqrt{\bar{\eta}_{s}}$. Notice that the entries of $C$ coincide with the $c_{s, \nu}$. Set

$$
\bar{p}_{s} \triangleq 2^{-m_{W}} \sum_{x} \eta_{s,(x, 0)}^{\infty}, \quad \bar{p}_{s, \nu}^{+} \triangleq 2^{-m_{W}} \sum_{x \mid x_{\nu-m_{P}}=1} \eta_{s,(x, 0)}^{\infty}, \quad \bar{p}_{s, \nu}^{-} \triangleq 2^{-m_{W}} \sum_{x \mid x_{\nu-m_{P}}=-1} \eta_{s,(x, 0)}^{\infty} .
$$

Then $E_{s, \nu}=\sum_{\nu} \frac{\bar{p}_{s, \nu}^{+}-\bar{p}_{s, \nu}^{-}}{\bar{p}_{s}}$. Since $R$ is an orthogonal matrix, $d W=\left(d W_{s}\right)_{s \in\{1, \ldots, m\}}$ and $R^{\dagger} d W$ define similar Wiener processes. Thus, with replacing $d W$ by $R^{\dagger} d W$ in $(30,31)$, we have

$$
\begin{aligned}
& d \rho_{t}=-i\left[H, \rho_{t}\right] d t+\sum_{\xi} \mathcal{L}_{\xi}\left(\rho_{t}\right) d t+\sum_{s}\left(\sum_{\nu} \frac{\bar{p}_{s, \nu}^{+}-\bar{p}_{s, \nu}^{-}}{\sqrt{\bar{p}_{s}}} \Lambda_{\nu}\left(\rho_{t}\right)\right) d W_{s}(t), \\
& d \rho_{t}^{e}=-i\left[H, \rho_{t}^{e}\right] d t+\sum_{\xi} \mathcal{L}_{\xi}\left(\rho_{t}^{e}\right) d t+\mathcal{K}\left(\rho_{t}, \rho_{t}^{e}\right) d t+\sum_{s} \sum_{\nu} \frac{\bar{p}_{s, \nu}^{+}-\bar{p}_{s, \nu}^{-}}{\sqrt{\bar{p}_{s}}} \Lambda_{\nu}\left(\rho_{t}^{e}\right) d W_{s}(t),
\end{aligned}
$$

with

$$
\mathcal{K}\left(\rho, \rho^{e}\right):=\sum_{s}\left(\sum_{\nu} \frac{\bar{p}_{s, \nu}^{+}-\bar{p}_{s, \nu}^{-}}{\bar{p}_{s}}\left(\operatorname{Tr}\left(\left(V_{\nu}+V_{\nu}^{\dagger}\right)\left(\rho-\rho^{e}\right)\right)\right)\right)\left(\sum_{\nu^{\prime}}\left(\bar{p}_{s, \nu^{\prime}}^{+}-\bar{p}_{s, \nu^{\prime}}^{-}\right) \Lambda_{\nu^{\prime}}\left(\rho^{e}\right)\right) .
$$

The infinitesimal generator associated to the Markov process $\left(\rho_{n}, \rho_{n}^{e}\right)$ satisfying dynamics $(24)$ and (25), can be written as follows

$$
\mathcal{A}_{n} f\left(\rho, \rho^{e}\right) \approx n \sum_{s} p_{s}(\rho)\left(f\left(\frac{\mathcal{M}_{s}^{n}(\rho)}{p_{s}(\rho)}, \frac{\mathcal{M}_{s}^{n}\left(\rho^{e}\right)}{p_{s}\left(\rho^{e}\right)}\right)-f\left(\rho, \rho^{e}\right)\right) .
$$


We can approximate the generator $\mathcal{A}_{n}$ via the second order expansion of $f$ around $\rho$ and $\rho^{e}$.

$$
\begin{aligned}
\mathcal{A}_{n} f\left(\rho, \rho^{e}\right)=n D_{\left(\rho, \rho^{e}\right)} f \cdot\left(\sum_{s} \mathcal{M}_{s}^{n}(\rho)-p_{s}(\rho) \rho, \sum_{s}\left(\mathcal{M}_{s}^{n}\left(\rho^{e}\right)-p_{s}\left(\rho^{e}\right) \rho^{e}\right) \frac{p_{s}(\rho)}{p_{s}\left(\rho^{e}\right)}\right) \\
\quad+n \sum_{s} p_{s}(\rho) \frac{1}{2} D_{\left(\rho, \rho^{e}\right)}^{2} f \cdot\left(\frac{\mathcal{M}_{s}^{n}(\rho)}{p_{s}(\rho)}-\rho \frac{\mathcal{M}_{s}^{n}\left(\rho^{e}\right)}{p_{s}\left(\rho^{e}\right)}-\rho^{e} ; \frac{\mathcal{M}_{s}^{n}(\rho)}{p_{s}(\rho)}-\rho, \frac{\mathcal{M}_{s}^{n}\left(\rho^{e}\right)}{p_{s}\left(\rho^{e}\right)}\right)+\mathcal{O}\left(n^{-1 / 2}\right) .
\end{aligned}
$$

Let us first calculate the first term in above. By using (10), (11) and (12), we find

$$
\begin{aligned}
\mathcal{M}_{s}^{n}(\rho)-p_{s}(\rho) \rho & =\sum_{x} \eta_{s,(x, 0)}^{n}\left(M_{x, 0} \rho M_{x, 0}^{\dagger}-p_{x, 0}(\rho) \rho\right)+\sum_{x, \mu} \eta_{s,(x, \mu)}^{n}\left(M_{x, \mu} \rho M_{x, \mu}^{\dagger}-p_{x, \mu}(\rho) \rho\right) \\
& =\sum_{x} \frac{\eta_{s,(x, 0)}^{n}{ }^{c_{W}}}{\sqrt{n}} \sum_{\nu} x_{\nu-m_{P}} \Lambda_{\nu}(\rho) \\
& +\sum_{x} \frac{\eta_{s,(x, 0)}^{n} c_{W}}{n}\left(-i[H, \rho]+\sum_{\xi} \mathcal{L}_{\xi}(\rho)-\sum_{\mu} \Upsilon_{\mu}(\rho) \operatorname{Tr}\left(V_{\mu} \rho V_{\mu}^{\dagger}\right)\right) \\
& +\sum_{x, \mu} \frac{\eta_{s,(x, \mu)}^{n}{ }^{c_{W}}}{n} \Upsilon_{\mu}(\rho) \operatorname{Tr}\left(V_{\mu} \rho V_{\mu}^{\dagger}\right) .
\end{aligned}
$$

We have

$$
\sum_{s} \mathcal{M}_{s}^{n}(\rho)-p_{s}(\rho) \rho=\frac{1}{n}\left(-i[H, \rho]+\sum_{\xi} \mathcal{L}_{\xi}(\rho)\right),
$$

since for all $(x, 0)$ and $(x, \mu): \sum_{s} \eta_{s,(x, 0)}^{n}=\sum_{s} \eta_{s,(x, \mu)}^{n}=1$. Also note that for all $\nu$ :

$$
\sum_{x} x_{\nu-m_{P}} \Lambda_{\nu}(\rho)=0
$$

Equation (35) can be rewritten as follows,

$$
\begin{aligned}
\mathcal{M}_{s}^{n}(\rho)-p_{s}(\rho) \rho & =\sum_{x} \frac{\eta_{s,(x, 0)}^{n}{ }^{c} W}{\sqrt{n}} \sum_{\nu} x_{\nu-m_{P}} \Lambda_{\nu}(\rho)+\mathcal{O}\left(n^{-1 / 2}\right) \\
& =\sum_{\nu} \frac{\bar{p}_{s, \nu}^{+}-\bar{p}_{s, \nu}^{-}}{\sqrt{n}} \Lambda_{\nu}(\rho)+\mathcal{O}\left(n^{-1 / 2}\right) .
\end{aligned}
$$

Thus $\mathcal{M}_{s}^{n}(\rho)-p_{s}(\rho) \rho=\mathcal{O}\left(n^{-1 / 2}\right)$. Now using (11) and (12), we have

$$
\begin{aligned}
p_{s}(\rho)= & \sum_{x} \eta_{s,(x, 0)}^{n} p_{x, 0}(\rho)+\sum_{x, \mu} \eta_{s,(x, \mu)}^{n} p_{x, \mu}(\rho)= \\
& \sum_{x} \eta_{s,(x, 0)}^{n} c_{W}\left(1+\frac{1}{\sqrt{n}} \sum_{\nu} x_{\nu-m_{P}} \operatorname{Tr}\left(\left(V_{\nu}+V_{\nu}^{\dagger}\right) \rho\right)-\right. \\
& \left.\frac{1}{n} \sum_{\mu} \operatorname{Tr}\left(\rho V_{\mu}^{\dagger} V_{\mu}\right)\right) \\
& +\sum_{x, \mu} \frac{\eta_{s,(x, \mu}^{n} c_{W}}{n} \operatorname{Tr}\left(V_{\mu} \rho V_{\mu}^{\dagger}\right) .
\end{aligned}
$$


By equation in above, the approximate value of $\frac{p_{s}(\rho)}{p_{s}\left(\rho^{e}\right)}$ is given by

$$
\begin{aligned}
\frac{p_{s}(\rho)}{p_{s}\left(\rho^{e}\right)} & =1+\frac{c_{W}}{\sqrt{n} \bar{p}_{s}} \sum_{x} \eta_{s,(x, 0)}^{n}\left(\sum_{\nu} x_{\nu-m_{P}}\left(\operatorname{Tr}\left(\left(V_{\nu}+V_{\nu}^{\dagger}\right) \rho\right)-\operatorname{Tr}\left(\left(V_{\nu}+V_{\nu}^{\dagger}\right) \rho^{e}\right)\right)\right)+\mathcal{O}\left(n^{-1 / 2}\right) \\
& =1+\frac{1}{\sqrt{n} \bar{p}_{s}}\left(\sum_{\nu}\left(\bar{p}_{s, \nu}^{+}-\bar{p}_{s, \nu}^{-}\right)\left(\operatorname{Tr}\left(\left(V_{\nu}+V_{\nu}^{\dagger}\right) \rho\right)-\operatorname{Tr}\left(\left(V_{\nu}+V_{\nu}^{\dagger}\right) \rho^{e}\right)\right)\right)+\mathcal{O}\left(n^{-1 / 2}\right) .
\end{aligned}
$$

Finally, we find the following

$$
\sum_{s} n\left(\mathcal{M}_{s}^{n}\left(\rho^{e}\right)-p_{s}\left(\rho^{e}\right) \rho^{e}\right) \frac{p_{s}(\rho)}{p_{s}\left(\rho^{e}\right)}=-i\left[H, \rho^{e}\right]+\sum_{\xi} \mathcal{L}_{\xi}\left(\rho^{e}\right)+\mathcal{K}\left(\rho, \rho^{e}\right)+\mathcal{O}\left(n^{-1 / 2}\right) .
$$

Since $p_{s}(\rho)=\bar{p}_{s}+\mathcal{O}\left(n^{-1 / 2}\right)$ and $\frac{\mathcal{M}_{s}^{n}(\rho)}{p_{s}(\rho)}-\rho=\mathcal{O}\left(n^{-1 / 2}\right)$, the zero order terms of

$$
n \sum_{s} p_{s}(\rho) \frac{1}{2} D_{\left(\rho, \rho^{e}\right)}^{2} f \cdot\left(\frac{\mathcal{M}_{s}^{n}(\rho)}{p_{s}(\rho)}-\rho, \frac{\mathcal{M}_{s}^{n}\left(\rho^{e}\right)}{p_{s}\left(\rho^{e}\right)}-\rho^{e} ; \frac{\mathcal{M}_{s}^{n}(\rho)}{p_{s}(\rho)}-\rho, \frac{\mathcal{M}_{s}^{n}\left(\rho^{e}\right)}{p_{s}\left(\rho^{e}\right)}-\rho^{e}\right)
$$

is given by the following computations:

$$
\begin{aligned}
& n \sum_{s} p_{s}(\rho) D_{\left(\rho, \rho^{e}\right)}^{2} f \cdot\left(\frac{\mathcal{M}_{s}^{n}(\rho)}{p_{s}(\rho)}-\rho, \frac{\mathcal{M}_{s}^{n}\left(\rho^{e}\right)}{p_{s}\left(\rho^{e}\right)}-\rho^{e} ; \frac{\mathcal{M}^{n}(\rho)}{p_{s}(\rho)}-\rho, \frac{\mathcal{M}_{s}^{n}\left(\rho^{e}\right)}{p_{s}\left(\rho^{e}\right)}-\rho^{e}\right) \\
& =n \sum_{s} \frac{1}{\bar{p}_{s}} D_{\left(\rho, \rho^{e}\right)}^{2} f \cdot\left(\mathcal{M}_{s}^{n}(\rho)-p_{s}(\rho) \rho, \mathcal{M}_{s}^{n}\left(\rho^{e}\right)-p_{s}\left(\rho^{e}\right) \rho^{e} ; \mathcal{M}_{s}^{n}(\rho)-p_{s}(\rho) \rho, \mathcal{M}_{s}^{n}\left(\rho^{e}\right)-p_{s}\left(\rho^{e}\right) \rho^{e}\right)+\mathcal{O}\left(n^{-1 / 2}\right) \\
& =\sum_{s} \frac{1}{\bar{p}_{s}} D_{\left(\rho, \rho^{e}\right)}^{2} f \cdot\left(\sum_{\nu}\left(\bar{p}_{s, \nu}^{+}-\bar{p}_{s, \nu}^{-}\right) \Lambda_{\nu}(\rho), \sum_{\nu}\left(\bar{p}_{s, \nu}^{+}-\bar{p}_{s, \nu}^{-}\right) \Lambda_{\nu}\left(\rho^{e}\right) ; \sum_{\nu^{\prime}}\left(\bar{p}_{s, \nu^{\prime}}^{+}-\bar{p}_{s, \nu^{\prime}}^{-}\right) \Lambda_{\nu^{\prime}}(\rho), \sum_{\nu^{\prime}}\left(\bar{p}_{s, \nu^{\prime}}^{+}-\bar{p}_{s, \nu^{\prime}}^{-}\right) \Lambda_{\nu^{\prime}}\left(\rho^{e}\right)\right) \\
& +\mathcal{O}\left(n^{-1 / 2}\right),
\end{aligned}
$$

where we have used Equation (37) and the fact that we have to keep only the zero order terms of $\frac{1}{p_{s}(\rho)}$ and $\frac{p_{s}(\rho)}{p_{s}\left(\rho^{e}\right)^{2}}$ which is equal to $\frac{1}{\bar{p}_{s}}$.

We get finally the following expression for the generator $\mathcal{A}$ which is given as the limit of $\mathcal{A}_{n}$ when $n$ goes to infinity.

$$
\begin{aligned}
& \mathcal{A} f\left(\rho, \rho^{e}\right)=D_{\rho} f \cdot\left(-i[H, \rho]+\sum_{\xi} \mathcal{L}_{\xi}(\rho),-i\left[H, \rho^{e}\right]+\sum_{\xi} \mathcal{L}_{\xi}\left(\rho^{e}\right)+\mathcal{K}\left(\rho, \rho^{e}\right)\right) \\
& +\frac{1}{2} \sum_{s} D_{\left(\rho, \rho^{e}\right)}^{2} f \cdot\left(\sum_{\nu} \frac{\bar{p}_{s, \nu}^{+}-\bar{p}_{s, \nu}^{-}}{\sqrt{\overline{p_{s}}}} \Lambda_{\nu}(\rho), \sum_{\nu} \frac{\bar{p}_{s, \nu}^{+}-\bar{p}_{s, \nu}^{-}}{\sqrt{\bar{p}_{s}}} \Lambda_{\nu}\left(\rho^{e}\right) ; \sum_{\nu^{\prime}} \frac{\bar{p}_{s, \nu^{\prime}}^{+}-\bar{p}_{s, \nu^{\prime}}^{-}}{\sqrt{\overline{p_{s}}}} \Lambda_{\nu^{\prime}}(\rho), \sum_{\nu^{\prime}} \frac{\bar{p}_{s, \nu^{\prime}}^{+}-\bar{p}_{s, \nu^{\prime}}^{-}}{\sqrt{\overline{p_{s}}}} \Lambda_{\nu^{\prime}}\left(\rho^{e}\right)\right) .
\end{aligned}
$$

The expression given in above corresponds well to the infinitesimal generator associated to the Markov process $\left(\rho, \rho^{e}\right)$ satisfying dynamics (33) and (34).

Comparing this equation to the one given in (2) and (5) for perfect measurements, we observe that the Poisson processes have completely disappeared. It just remains the ensemble average $\sum_{\mu} \mathcal{L}_{\mu}(\rho)$ in the deterministic part. Also, the original $m_{W}$ Wiener processes indexed by $\nu$ have been reorganized in $m$ Wiener processes indexed by $s$. 


\subsubsection{Proof of Theorem 4 when $S^{P}=\{1, \ldots, m\}$ and $S^{W}=\varnothing$}

In this case, we have

$$
\begin{aligned}
& d \rho_{t}=-i\left[H, \rho_{t}\right] d t+\sum_{\xi} \mathcal{L}_{\xi}\left(\rho_{t}\right) d t \\
+ & \sum_{s}\left(\frac{\bar{\theta}_{s} \rho_{t}+\sum_{\mu} \bar{\eta}_{s, \mu} V_{\mu} \rho_{t} V_{\mu}^{\dagger}}{\bar{\theta}_{s}+\sum_{\mu} \bar{\eta}_{s, \mu} \operatorname{Tr}\left(V_{\mu} \rho_{t} V_{\mu}^{\dagger}\right)}-\rho_{t}\right)\left(d N_{s}(t)-\left(\bar{\theta}_{s}+\sum_{\mu} \bar{\eta}_{s, \mu} \operatorname{Tr}\left(V_{\mu} \rho_{t} V_{\mu}^{\dagger}\right)\right) d t\right),
\end{aligned}
$$

and

$$
\begin{aligned}
& d \rho_{t}^{e}=-i\left[H, \rho_{t}^{e}\right] d t+\sum_{\xi} \mathcal{L}_{\xi}\left(\rho_{t}^{e}\right) d t \\
+ & \sum_{s}\left(\frac{\bar{\theta}_{s} \rho_{t}^{e}+\sum_{\mu} \bar{\eta}_{s, \mu} V_{\mu} \rho_{t}^{e} V_{\mu}^{\dagger}}{\bar{\theta}_{s}+\sum_{\mu} \bar{\eta}_{s, \mu} \operatorname{Tr}\left(V_{\mu} \rho_{t}^{e} V_{\mu}^{\dagger}\right)}-\rho_{t}^{e}\right)\left(d N_{s}(t)-\left(\bar{\theta}_{s}+\sum_{\mu} \bar{\eta}_{s, \mu} \operatorname{Tr}\left(V_{\mu} \rho_{t}^{e} V_{\mu}^{\dagger}\right)\right) d t\right) .
\end{aligned}
$$

The infinitesimal generator associated to the Markov process $\left(\rho_{n}, \rho_{n}^{e}\right)$ is given by the following

$$
\mathcal{A}_{n} f\left(\rho, \rho^{e}\right) \approx n \sum_{s} p_{s}(\rho)\left(f\left(\frac{\mathcal{M}_{s}^{n}(\rho)}{p_{s}(\rho)}, \frac{\mathcal{M}_{s}^{n}\left(\rho^{e}\right)}{p_{s}\left(\rho^{e}\right)}\right)-f\left(\rho, \rho^{e}\right)\right) .
$$

The equation in above can be rewritten as follows

$$
\begin{aligned}
\mathcal{A}_{n} f\left(\rho, \rho^{e}\right) \approx n \sum_{s} p_{s}(\rho)\left(f\left(\frac{\mathcal{M}_{s}^{n}(\rho)}{p_{s}(\rho)}, \frac{\mathcal{M}_{s}^{n}\left(\rho^{e}\right)}{p_{s}\left(\rho^{e}\right)}\right)-f\left(\rho, \rho^{e}\right)\right) \\
+n D_{\left(\rho, \rho^{e}\right)} f \cdot\left(\sum_{s}\left(\mathcal{M}_{s}^{n}(\rho)-p_{s}(\rho) \rho\right), \sum_{s}\left(\mathcal{M}_{s}^{n}\left(\rho^{e}\right)-p_{s}\left(\rho^{e}\right) \rho^{e}\right)+\mathcal{K}_{2}\left(\rho, \rho^{e}\right)\right) \\
\quad-n \sum_{s} p_{s}(\rho) D_{\left(\rho, \rho^{e}\right)} f \cdot\left(\frac{\mathcal{M}_{s}^{n}(\rho)}{p_{s}(\rho)}-\rho, \frac{\mathcal{M}_{s}^{n}\left(\rho^{e}\right)}{p_{s}\left(\rho^{e}\right)}-\rho^{e}\right),
\end{aligned}
$$

with $\mathcal{K}_{2}\left(\rho, \rho^{e}\right):=\sum_{s}\left(\frac{\mathcal{M}_{s}^{n}\left(\rho^{e}\right)}{p_{s}\left(\rho^{e}\right)}-\rho^{e}\right)\left(p_{s}(\rho)-p_{s}\left(\rho^{e}\right)\right)$.

Now let us give the expression of $\mathcal{M}_{s}^{n}(\rho)$

$$
\begin{aligned}
\mathcal{M}_{s}^{n}(\rho) & =\sum_{x} \eta_{s,(x, 0)}^{n} M_{x, 0} \rho M_{x, 0}^{\dagger}+\sum_{x, \mu} \eta_{s,(x, \mu)}^{n} M_{x, \mu} \rho M_{x, \mu}^{\dagger} \\
= & \frac{1}{n}\left(\bar{\theta}_{s} \rho+\sum_{\mu} \bar{\eta}_{s, \mu} V_{\mu} \rho V_{\mu}^{\dagger}\right)+\mathcal{O}\left(n^{-\frac{3}{2}}\right),
\end{aligned}
$$

where for the last term in above, we have used (10), (12), (23), $\bar{\theta}_{s}=c_{W} \sum_{x} \widetilde{\eta}_{s,(x, 0)}$ and $\bar{\eta}_{s, \mu}=c_{W} \sum_{x} \eta_{s,(x, \mu)}^{\infty}$.

Consequently, the probability $p_{s}(\rho)$ has the following form

$$
p_{s}(\rho)=\frac{1}{n}\left(\bar{\theta}_{s}+\sum_{\mu} \bar{\eta}_{s, \mu} \operatorname{Tr}\left(V_{\mu} \rho V_{\mu}^{\dagger}\right)\right)+\mathcal{O}\left(n^{-\frac{3}{2}}\right) .
$$


Now by using Equations (36), (41) and (42), we find the following expression for the limit of the generator $\mathcal{A}_{n}$ expressed in (40)

$$
\begin{aligned}
& \mathcal{A} f\left(\rho, \rho^{e}\right)=D_{\left(\rho, \rho^{e}\right)} f \cdot\left(-i[H, \rho]+\sum_{\xi} \mathcal{L}_{\xi}(\rho),-i\left[H, \rho^{e}\right]+\sum_{\xi} \mathcal{L}_{\xi}\left(\rho^{e}\right)+\mathcal{K}_{2}\left(\rho, \rho^{e}\right)\right) \\
& +\sum_{s}\left(\bar{\theta}_{s}+\sum_{\mu} \bar{\eta}_{s, \mu} \operatorname{Tr}\left(V_{\mu} \rho V_{\mu}^{\dagger}\right)\right) \times \\
& \left(f\left(\frac{\bar{\theta}_{s} \rho+\sum_{\mu} \bar{\eta}_{s, \mu} V_{\mu} \rho V_{\mu}^{\dagger}}{\bar{\theta}_{s}+\sum_{\mu} \bar{\eta}_{s, \mu} \operatorname{Tr}\left(V_{\mu} \rho V_{\mu}^{\dagger}\right)}, \frac{\bar{\theta}_{s} \rho^{e}+\sum_{\mu} \bar{\eta}_{s, \mu} V_{\mu} \rho^{e} V_{\mu}^{\dagger}}{\bar{\theta}_{s}+\sum_{\mu} \bar{\eta}_{s, \mu} \operatorname{Tr}\left(V_{\mu} \rho^{e} V_{\mu}^{\dagger}\right)}\right)-f\left(\rho, \rho^{e}\right)\right) \\
& -\sum_{s}\left(\bar{\theta}_{s}+\sum_{\mu} \bar{\eta}_{s, \mu} \operatorname{Tr}\left(V_{\mu} \rho V_{\mu}^{\dagger}\right)\right) \times \\
& D_{\left(\rho, \rho^{e}\right)} f \cdot\left(\frac{\bar{\theta}_{s} \rho+\sum_{\mu} \bar{\eta}_{s, \mu} V_{\mu} \rho V_{\mu}^{\dagger}}{\bar{\theta}_{s}+\sum_{\mu} \bar{\eta}_{s, \mu} \operatorname{Tr}\left(V_{\mu} \rho V_{\mu}^{\dagger}\right)}-\rho, \frac{\bar{\theta}_{s} \rho^{e}+\sum_{\mu} \bar{\eta}_{s, \mu} V_{\mu} \rho^{e} V_{\mu}^{\dagger}}{\bar{\theta}_{s}+\sum_{\mu} \bar{\eta}_{s, \mu} \operatorname{Tr}\left(V_{\mu} \rho^{e} V_{\mu}^{\dagger}\right)}-\rho^{e}\right) .
\end{aligned}
$$

In fact, we have

$$
\mathcal{A}_{n} f\left(\rho, \rho^{e}\right)=\mathcal{A} f\left(\rho, \rho^{e}\right)+\mathcal{O}\left(n^{-\frac{1}{2}}\right) .
$$

We observe that the expression of $\mathcal{A} f\left(\rho, \rho^{e}\right)$ corresponds well to the generator associated to the Markov process $\left(\rho, \rho^{e}\right)$ satisfying SMEs (38) and (39). Now use the tightness property of the sequence $\left(\rho_{n}, \rho_{n}^{e}\right)$ to conclude the convergence of the processes $\left(\rho_{n}, \rho_{n}^{e}\right)$ towards $\left(\rho, \rho^{e}\right)$.

\subsubsection{Proof of Theorem 4 when $S^{P} \neq \varnothing$ and $S^{W} \neq \varnothing$}

In this general case, the Markov generator of $\left(\rho_{n}, \rho_{n}^{e}\right)$ is decomposed into two different sums:

$$
\begin{aligned}
& \mathcal{A}_{n} f\left(\rho, \rho^{e}\right) \approx n \sum_{s \in\{1, \ldots, m\}} p_{s}(\rho)\left(f\left(\frac{\mathcal{M}_{s}^{n}(\rho)}{p_{s}(\rho)}, \frac{\mathcal{M}_{s}^{n}\left(\rho^{e}\right)}{p_{s}\left(\rho^{e}\right)}\right)-f\left(\rho, \rho^{e}\right)\right) \\
= & n \sum_{s \in S^{W}} p_{s}(\rho)\left(f\left(\frac{\mathcal{M}_{s}^{n}(\rho)}{p_{s}(\rho)}, \frac{\mathcal{M}_{s}^{n}\left(\rho^{e}\right)}{p_{s}\left(\rho^{e}\right)}\right)-f\left(\rho, \rho^{e}\right)\right)+n \sum_{s \in S^{P}} p_{s}(\rho)\left(f\left(\frac{\mathcal{M}_{s}^{n}(\rho)}{p_{s}(\rho)}, \frac{\mathcal{M}_{s}^{n}\left(\rho^{e}\right)}{p_{s}\left(\rho^{e}\right)}\right)-f\left(\rho, \rho^{e}\right)\right) .
\end{aligned}
$$

The limit for $n$ large of each sum versus $s \in S^{W}$ and $s \in S^{P}$ is then obtained following exactly the same calculations as the ones given in subsections 3.3.1 and 3.3.2. Since $(30,31)$ are just the concatenation of $(33,34)$ for the Wiener part and $(38,39)$ for the Poisson part, we get directly the convergence of the processes $\left(\rho_{n}(t), \rho_{n}^{e}(t)\right)$ towards $\left(\rho_{t}, \rho_{t}^{e}\right)$.

\section{Conclusion}

For a large class of SMEs driven by Wiener and Poisson processes, Theorem 5 shows that $\operatorname{Tr}^{2}\left(\sqrt{\sqrt{\rho}} \rho^{e} \sqrt{\rho}\right)$ between the quantum state $\rho_{t}$ and its estimate $\rho_{t}^{e}$ is a submartingale. Thus the "metric" $1-\operatorname{Tr}^{2}\left(\sqrt{\sqrt{\rho} \rho^{e} \sqrt{\rho}}\right)$ is a non-negative super-martingale that vanishes only when $\rho=\rho^{e}$. A natural question is the following: do there exist other "metrics" $D\left(\rho, \rho^{e}\right)$ that are super-martingales for such a large class of quantum systems?

Any such "metric" $D$ must be contractive for all Lindblad equations: in Theorem 5 , there is no restriction on the degree of incompleteness of the measurements. Thus we can assume 
$\bar{\eta}_{s}=0, S^{W}=\{1, \ldots, m\}$ and $S^{P}=\emptyset$. In this case, $\rho$ and $\rho^{e}$ obey the same ordinary Lindblad differential equation

$$
\frac{d}{d t} \rho=-i[H, \rho]+\sum_{\xi} V_{\xi} \rho V_{\xi}^{\dagger}-\frac{1}{2}\left(V_{\xi}^{\dagger} V_{\xi} \rho+\rho V_{\xi}^{\dagger} V_{\xi}\right)
$$

where $H$ and $V_{\xi}$ are arbitrary. In [20], Petz has given, via the theory of operator monotone functions, a complete characterization of distance that are contractive for all Lindblad evolutions. Could we exploit Petz results to characterize "metrics" $D\left(\rho, \rho^{e}\right)$ that are supermartingale for all the quantum filtering processes considered in this paper?

\section{References}

[1] H. Amini. Stabilization of Discrete-Time Quantum Systems and Stability of ContinuousTime Quantum Filters. PhD thesis, Mines ParisTech, 2012.

[2] H. Amini, M. Mirrahimi, and P. Rouchon. On stability of continuous-time quantumfilters. In Proceedings of the 50th IEEE Conference on Decision and Control, pages 6242-6247, 2011.

[3] Stéphane Attal and Yan Pautrat. From repeated to continuous quantum interactions. In Annales Henri Poincaré, volume 7, pages 59-104. Springer, 2006.

[4] A. Barchielli and V. P. Belavkin. Measurements continuous in time and a posteriori states in quantum mechanics. Journal of Physics A: Mathematical and General, 24(7):1495, 1991.

[5] A. Barchielli and M. Gregoratti. Quantum Trajectories and Measurements in Continuous Time: the Diffusive Case, volume 782. Springer Verlag, 2009.

[6] Benoist T. Bauer, M. and D. Bernard. Repeated quantum non-demolition measurements: Convergence and continuous time limit. Annales Henri Poincaré, 14(4):639679, 2013.

[7] V. P. Belavkin. Quantum filtering of Markov signals with white quantum noise. Radiotechnika i Electronika, 25:1445-1453, 1980.

[8] V. P. Belavkin. Quantum stochastic calculus and quantum nonlinear filtering. Journal of Multivariate Analysis, 42(2):171-201, 1992.

[9] V.P. Belavkin. Eventum mechanics of quantum trajectories: Continual measurements, quantum predictions and feedback control. arXiv:math-ph/0702079, 2007.

[10] T Benoist and C. Pellegrini. Large time behavior and convergence rate for quantum filters under standard non demolition conditions. Communications in Mathematical Physics, in press, 2013.

[11] V. B. Braginsky and F. Y Khalili. Quantum Measurement. Cambridge Univ Pr, 1995.

[12] H. . Carmichael. An Open Systems Approach to Quantum Optics. Springer-Verlag, 1993. 
[13] J. Dalibard, Y. Castin, and K. Mølmer. Wave-function approach to dissipative processes in quantum optics. Phys. Rev. Lett., 68(5):580-583, 1992.

[14] E. B. Davies. Quantum theory of open systems. 1976.

[15] J. Gough and A.i Sobolev. Stochastic Schrödinger equations as limit of discrete filtering. Open Systems \& Information Dynamics, 11(03):235-255, 2004.

[16] S. Haroche and J.-M. Raimond. Exploring the Quantum: Atoms, Cavities and Photons. Oxford University Press, New York, 2006.

[17] C. Pellegrini. Existence, uniqueness and approximation for stochastic Schrödinger equation: the poisson case. arXiv preprint arXiv:0709.3713, 2007.

[18] C. Pellegrini. Existence, uniqueness and approximation of a stochastic Schrödinger equation: the diffusive case. The Annals of Probability, pages 2332-2353, 2008.

[19] C. Pellegrini. Markov chains approximation of jump-diffusion stochastic master equations. Annales de l'Institut Henri Poincaré, Probabilités et Statistiques, 46(4):924-948, 2010 .

[20] D. Petz. Monotone metrics on matrix spaces. Linear Algebra and its Applications, 244:81-96, 1996.

[21] P. Rouchon. Fidelity is a sub-martingale for discrete-time quantum filters. IEEE Transactions on Automatic Control, 56(11):2743-2747, 2011.

[22] C. Sayrin, I. Dotsenko, X. Zhou, B. Peaudecerf, T. Rybarczyk, S. Gleyzes, P. Rouchon, M. Mirrahimi, H. Amini, M. Brune, J.-M. Raimond, and S. Haroche. Real-time quantum feedback prepares and stabilizes photon number states. Nature, 477(7362):73-77, 2011.

[23] A. Somaraju, I. Dotsenko, C. Sayrin, and P. Rouchon. Design and stability of discretetime quantum filters with measurement imperfections. In Proceedings of American Control Conference, pages 5084-5089, 2012.

[24] R. van Handel. Filtering, Stability, and Robustness. PhD thesis, California Institute of Technology, 2006.

[25] R. van Handel. The stability of quantum Markov filters. Infinite Dimensional Analysis, Quantum Probability and Related Topics, 12(1):153-172, 2009.

[26] H. M. Wiseman and G. J. Milburn. Quantum Measurement and Control. Cambridge University Press, 2009. 